BULL. AUSTRAL. MATH. SOC.

06D30, 06005, 08B20, 08B30

VOL. $24(1981),|6|-203$.

(03860)

\title{
DISTRIBUTIVE OCKHAM ALGEBRAS: FREE ALGEBRAS AND INJECTIVITY
}

\author{
Moshe S. GOLdBerg
}

\begin{abstract}
This paper centres around the variety 0 of distributive Ockham algebras, and those subvarieties of 0 which are generated by a single finite subdirectly irreducible algebra $A$. We use H.A. Priestley's duality for bounded distributive lattices throughout. First, intrinsic descriptions of the duals of certain finite subdirectly irreducibles are given; these are later used to determine projectives in the dual categories. Next, left adjoints to the forgetful functors from 0 and $\operatorname{var}(A)$ into bounded distributive lattices are obtained, thereby allowing us to describe all free algebras and coproducts of arbitrary algebras. Finally, by applying the duality, we characterize injectivity in $\operatorname{Var}(A)$ for each finite subdirectly irreducible algebra $A$.
\end{abstract}

De Morgan algebras, natural generalizations of Boolean algebras were introduced by Moisil [19] and have since been extensively studied by various authors including Kalman [18] who called them i-lattices, Bialynicki-Birula and Rasiowa [4], [5], [6], who called them quasi-Boolean lattices, and Monteiro [20]. For a summary of the basic results see [1]. The study of distributive Ockham algebras, a further generalization, was commenced by Berman [3] and contined by Urquhart [23] who named them Ockham lattices and described the subdirectly irreducible and free algebras. A far reaching generalization has also been undertaken by Cornish [8], but

Received 2 March 1981. 
is as yet in manuscript form.

In this paper, we carry on the work of Urquhart. In Section 1, after giving the basic definitions and notation, we outline Priestley's duality for bounded distributive lattices. Throughout the rest of the paper we repeatedly make use of the duality to study certain properties in the variety of distributive Ockham algebras and in those subvarieties which are generated by finite algebras. In Section 2 we give an equational description of these varieties and discuss their subdirectly irreducibles. In Section 3 a more general description of the free algebras is given by considering the category of distributive Ockham algebras as a reflective subcategory of bounded distributive lattices. This also allows us to determine coproducts in this category, thus expanding a theorem due to Berman and Dwinger [1, Theorem 2, p. 216]. In Section 4 we use our description of the subdirectly irreducibles to find the injective algebras in each of the subvarieties mentioned above; our descriptions are obtained via a well-known theorem of Day [12], which links the injectives to the subdirectly irreducibles under certain conditions. Our results generalize the characterization of injective De Morgan algebras given by Cignoli in [7].

\section{Prel iminarjes}

An Ockham algebra $\langle A ; \vee, \wedge, \sim, 0,1\rangle$ is an algebra of type $\langle 2,2,1,0,0\rangle$, where $\langle A ; \vee, \wedge, 0,1\rangle$ is a bounded lattice, and $\sim$ is a dual endomorphism of $A$; that is, the equations

$$
\sim(x \wedge y)=\sim x \vee \sim y, \sim(x \vee y)=\sim x \wedge \sim y, \sim 0=1, \sim 1=0,
$$

hold identically. The class of distributive Ockham algebras is a variety, which will be denoted by 0 .

Priestley's duality for bounded distributive lattices was developed in [21] and [22].

Let $(X ; \tau ; \leq)$ be an ordered topological space; that is $(X, \tau)$ is a topological space and $\leq$ is a partial order on $X$. A subset $U$ of $X$ is called an order-ideal (dual order-ideal) if for all $x, y$ in $X$, $x \leq y$ and $y \in U(x \in U)$ implies $x \in U(y \in U)$. We say that $(X ; \tau ; \leq)$ is totally order-disconnected if for all $x, y$ in $X$, whenever $x \neq y$ there exists a clopen order-ideal $U$ of $X$, such that $x \in U$ and 
$y \notin U$. In the sequel, we will simply refer to an ordered topological space $(X ; \tau ; \leq)$ as $X$.

Denote the category of bounded distributive lattices by $D$, and denote the category of compact totally order-disconnected spaces with continuous order-preserving maps by $P$. Priestley's duality states that $P$ and $D$ are dual categories: the set $O(X)$ of clopen order-ideals of an object $X$ in $P$ is closed under union and intersection, and so is an object of $D$; and for each lattice $A$ in $D$, an object of $P$ is obtained by endowing the poset $P(A)$ of prime ideals of $A$ with an appropriate topology. Furthermore, $A \cong O(P(A))$ and $X \cong P(O(X))$, via natural isomorphisms.

If $X$ is an object of $P$ and $g$ is a continuous order-reversing map on $X$, then $(X ; g)$ (henceforth simply referred to as $X$ ), is called an ockham space. We will denote by $S$ the category whose objects are Ockham spaces, and whose morphisms are continuous order-preserving maps which commute with $g$. In [23], it is shown that 0 and $S$ are dual categories. If $A$ is an object of 0 , then the map $g$ defined on $P(A)$ by

$$
g(x)=\{a \in A|\sim a| x\} \text {, for each } x \text { in } P(A) \text {, }
$$

is continuous and order-reversing; and if $X$ is an object of $S$, then we can define a dual endomorphism $\sim$ on $O(X)$ by setting

$$
\sim U=X-g^{+}(U) \text {, for each } U \text { in } O(X) \text {. }
$$

If $X \in S$, then a subset $Y$ of $X$ will be called a g-subset if $x \in Y$ implies $g(x) \in Y$. For a subset $Y$ of $X, g^{\omega}(Y)$ will denote the smallest $g$-subset containing $Y$, and the lattice of all closed g-subsets of $X$ will be denoted by $G(X)$. We note that since 0 and $P$ are contravariant functors, and 2 is injective in both $D$ and $P$, injections in each category correspond with surjections in the dual category. It is easily verified that if $f$ in $S(Y, X)$ is injective, then $Y$ is isomorphic to a closed $g$-subset of $X$. This leads to the following result, which is proved in [23].

THEOREM 1.1. If $A$ is a distributive Ockham algebra, then the lattice $\operatorname{Con}(A)$, of congruences on $A$, is dually isomorphic to $G(P(A))$. 


\section{Subvarieties and subdirectly irreducibles}

We will denote the usual class operators corresponding to isomorphic copies, homomorphic images, subalgebras, and direct products by I, H, S, and $P$, respectively; and $S i(K)$ will denote the class of subdirectly irreducible algebras in a class $K$. On the other hand if. $X$ is a class of Ockham spaces, then $G(X)$ and $M(X)$ will denote respectively the classes of closed $g$-subsets and $S$-morphic images of spaces in $X$. Finally, for a class $K$ of Ockham algebras, $\operatorname{Var}(K)$ will denote the smallest variety containing $K$.

We begin with some general results on subdirectly irreducibles, to which we shall repeatedly refer. The first two are due to Urquhart and can be found in [23].

THEOREM 2.1. If $A$ is a distributive Ockham algebra, then $A$ is subdirectly-irreducible if and only if there is an open subset $U$ of $P(A)$ such that $\overline{g^{\omega}(\{x\})}=P(A)$ for all $x$ in $U$.

COROLLARY 2.2. If $A$ is a finite distributive Ockham algebra, then $A$ is subdirectly irreducible if and only if $g^{\omega}(\{x\})=P(A)$ for some $x$ in $P(A)$.

Note that the element $x$ in Corollary 2.2 is not necessarily unique; in general, if $X$ is the dual of a finite subdirectly irreducible distributive Ockham algebra, then any element which generates $X$ under $g$ will be called an end of $X$. If $O(X)$ is not simple then it is clear that this element is unique, and it will be denoted by $e_{X}$ (or simply $e$ if no confusion arises). If $O(X)$ is simple, then the following two results show that every element of $X$ is an end, and in this case we shall always specify one particular element which will be denoted by $e_{X}$ (or e).

THEOREM 2.3. If $A$ is a distributive Ockham algebra, then $A$ is simple if and only if $\overline{g^{\omega}(\{x\})}=P(A)$ for all $x$ in $P(A)$.

Proof. Immediate from Theorem 1.1.

COROLLARY 2.4. If $A$ is a finite distributive Ockham algebra, then $A$ is simple if and only if $g^{\omega}(\{x\})=P(A)$ for all $x$ in $P(A)$. 
Let $A$ be a finite subdirectly irreducible distributive Ockham algebra. If $e$ is the end of $P(A)$ and $Y$ is a g-subset of $P(A)$, then it is clear that $g^{k}(e)$ is the end of $Y$, where $k=\min \left\{l \mid g^{l}(e) \in Y\right\}$; whence $O(Y)$ is subdirectly irreducible, we use this fact to obtain the following result.

PROPOSITION 2.5. Let $A$ be a finite set of finite subdirectly irreducible distributive Ockham algebras and let $\mathrm{K}=\operatorname{var}(\mathrm{A})$. Then the subdixectly irreducibles of $K$ are precisely all the homomorphic images of subalgebras of algebras in $A$.

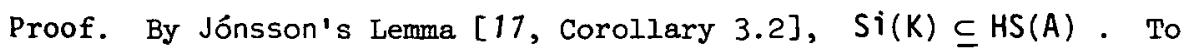
see the converse, let $B$ be a subalgebra of $A$, where $A \in A$. Then $P(B)$ is an S-morphic image (under $f$, say) of $P(A)$. Since $f$ is onto, it is clear that if $e$ is the end of $P(A)$, then $f(e)$ is the end of $P(B)$; whence $B$ is subdirectly irreducible. The rest follows from the remark preceding this proposition, which tells us that homomorphic images of finite subdirectly irreducible distributive Ockham algebras are themselves subdirectly irreducible.

COROLLARY 2.6. If the algebras in A of Proposition 2.5 are Boolean lattices, then the subdirectly irreducibles of $K$ are precisely all the subalgebras of algebras in A.

Proof. By Proposition 2.5 and Jónsson's Lemma, we have $\mathrm{S}(\mathrm{A}) \subseteq \mathrm{HS}(\mathrm{A})=\mathrm{Si}(\mathrm{K})$. To show the converse, let $Y$ be a closed $g$-subset of $X$, where $X$ is an S-morphic image (under $h$ ) of $P(A)$, for some $A$ in $A$. Let $e$ be the end of $P(A)$ and define $f: P(A)+Y$ by

$$
f(z)=g^{k} h(z) \text {, where } k=\min \left\{Z \in N \mid g^{2}(e) \in h^{+}(y)\right\} \text {. }
$$

It is easily seen that $f$ is well-defined and onto, since $Y$ is a $g$-subset of $X$; and $f$ commutes with $g$ since $h$ does. $P(A)$ is totally unordered because $A$ is Boolean, hence $f$ is also trivially order-preserving. Thus $Y$ is an $S$-morphic image of $P(A)$; so $H S(A) \subseteq S(A)$.

For $m \neq n, m, n \geq 0$, let $0_{m, n}$ denote the class of those distributive Ockham algebras whose dual spaces $X$ satisfy the equation 


$$
g^{m}(x) \leq g^{n}(x)
$$

Let $P_{m, n}=0_{m, n} \cap 0_{n, m}$, where $m>n$. The dual category of $P_{m, n}$ will be denoted by $S_{m, n}$.

Let $m-n$ be odd, and let $X$ be the dual of an algebra in $P_{m, n}$. Without loss of generality, suppose $m$ is odd and $n$ is even. Then for each $U$ in $O(X)$,

$$
\begin{aligned}
{\stackrel{m}{m} U \cap \sim^{n} U} & =\left[X-\left(g^{m}\right)^{+}(U)\right] \cap\left(g^{n}\right)^{+}(U) \\
& =\left[X-\left(g^{n}\right)^{+}(U)\right] \cap\left(g^{n}\right)^{+}(U) \\
& =\varnothing
\end{aligned}
$$

and

$$
\begin{aligned}
\sim_{U}^{m_{U}} \sim^{n} U & =\left[X-\left(g^{m}\right)^{+}(U)\right] \cup\left(g^{n}\right)^{+}(U) \\
& =\left[X-\left(g^{n}\right)^{+}(U)\right] \cup\left(g^{n}\right)^{+}(u) \\
& =X .
\end{aligned}
$$

Now, for $A$ in $\mathrm{P}_{\mathrm{m}, \mathrm{n}}, \sim^{m} a=\sim^{n}\left(\sim^{m-n_{a}}\right)$ for each $a$ in $A$, thus

whenever $m-n$ is odd, $\left\{\sim_{a}^{n} \mid a \in A\right\}$ is a Boolean algebra, and we have the following equational characterization of $P_{m, n}$.

PROPOSITION 2.7. Let $m>n, n \geq 0$, and let $A \in 0$. Then

(i). when $m-n$ is odd, $A \in \mathrm{P}_{\mathrm{m}, \mathrm{n}}$ if and only if $\sim^{m} a \wedge \sim^{n} a=0$ and $\sim_{a}^{m} \vee \sim^{n} a=1$ for each $a$ in $A$;

(ii) when $m-n$ is even, $A \in \mathrm{P}_{\mathrm{m}, \mathrm{n}}$ if and only if $\sim^{m} a=\sim^{n} a$ for each $a$ in $A$.

Note in particular that by Proposition 2.7 ( $i i), \mathrm{P}_{2,0}$ is the class of De Morgan algebras. The $P_{m, n}$ 's play an important rôle in the study of distributive Ockham algebras. For instance, if $A$ is a finite distributive Ockham algebra, then for each $x$ in $P(A)$, there exists a smallest pair $\left(m_{x}, n_{x}\right)$ of natural numbers, with $n_{x}<m_{x}$, such that 
$g^{m}(x)=g^{n} x(x)$. If we set $n=\max \left(n_{x} \mid x \in P(A)\right)$ and $m=n+1 . c \cdot m \cdot\left(m_{x^{-n} x} \mid x \in P(A)\right)$, we see that $g^{m}=g^{n}$ on $P(A)$. We have proved the following useful result.

PROPOSITION 2.8. Every finite distributive Ockham algebra is in $\mathrm{P}_{\mathrm{m}, \mathrm{n}}$ for some $n \geq 0, m>n$.

For $m>n$ and $n \geq 0$, let $m_{n}$ be the structure $\left(\mathbb{Z}_{m}, r_{n}\right)$ where $\gamma_{n}: \mathbb{Z}_{m} \rightarrow \mathbb{Z}_{m}$ is defined by $\gamma_{n}(k)=k+1$ for $0 \leq k<m-1$, and $r_{n}(m-1)=n$.

Let $L_{m, n}$ be the dual algebra of the Ockham space $m_{n}$ with the discrete topology and order, and $g=\gamma_{n}$. Let $L_{\omega}$ be the dual algebra of the Ockham space $B N$ the Stone-Čech compactification of the natural numbers with the discrete topology, where the order on $B N$ is discrete, and $g: B N \rightarrow P N$ is the unique extension of the successor map on $N$. For basic information about $B N$ we refer the reader to [15, chapter 6] and [24]. In [23], it is shown that $0=\operatorname{ISP}\left(L_{\omega}\right)$ and $P_{m, n}=\operatorname{ISP}\left(L_{m, n}\right)$. There, $L_{\omega}$ is actually defined to be $2^{\mathrm{N}}$, the set of all subsets of the natural numbers. But, it is quite easily seen that the two definitions are equivalent once it is recalled that $B$ is left adjoint to the forgetful functor, $|-|$ from Comp, the category of compact (Hausdorff) spaces, into Set; whence

$$
O(B N) \cong \operatorname{comp}(B N, 2) \cong \operatorname{set}(N,|2|) \cong 2^{N}
$$

Before generalizing from $P_{m, n}$ to any subvariety generated by a finite subdirectly irreducible distributive Ockham algebra we include some consequences of Proposition 2.8.

COROLLARY 2.9. (i) A finite distributive Ockham algebra is simple if and only if it is a subalgebra of $L_{m, 0}$ for some $m>0$.

(ii) A finite distributive Ockhan algebra is subdirectly irreducible if and only if it is a subalgebra of $L_{m, n}$ for some $m>n, n \geq 0$. 
Let $X$ be the dual of any finite subdirectly irreducible Ockham algebra. Let $(m, n)$ be the smallest element of $N \times \mathbf{N}$ such that $O(X)$ is a subalgebra of $L_{m, n}$. It is clear that $m$ must in fact be the cardinality of $X$. To simplify our notation, wherever possible we will denote $g^{k}\left(e_{X}\right)$ by $k$. Note that the non-empty $g$-subsets of $X$ are precisely the sets

$$
x_{k}=\{k, k+1, \ldots, m-1\} \text {, }
$$

where $k \in \mathbb{Z}_{n+1}$; thus $\operatorname{Con}(O(X))$ is an $n+2$-element chain. In particular, we have

PROPOSITION 2.10. For $m>n, n \geq 0, \operatorname{Con}\left(L_{m, n}\right)$ is an n+2-element chain.

The dual of a finite subdirectly irreducible distributive Ockham algebra may be viewed as in Figure 1, though it should be remembered that in this diagram, we have suppressed the order relation.

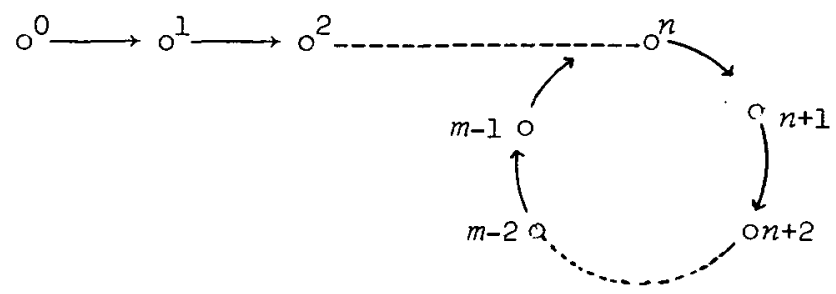

FIGURE 1

For the rest of this section, $X$ will be the dual of a finite subdirectly irreducible distributive Ockham algebra, with end $e$, $\operatorname{Card}(X)=m$, and $n=\min \left\{k \in \mathbb{Z}_{m} \mid g^{k}(e)=g^{m}(e)\right\}$. We will often talk about two particular subsets of $X: L=\{n, n+1, \ldots, m-1\}$ and $T=\{0,1, \ldots, n-1\}$. For obvious reasons, these will be called respectively the loop of $X$ and the tail of $X$. Note that $O(X)$ is 
simple if and only if $X$ is a loop; that is, if and only if the tail of $X$ is empty .

Let $K=\operatorname{HSP}(O(X))$; once again using Jónsson's Lemma, we obtain $K=\operatorname{ISPHS}(O(X))$. Recall that $K$ satisfies the congmence extension property if

(CEP): for each algebra $A$ in $K$, and every subalgebra $B$ of $A$, every congruence on $B$ is the restriction of some congruence on $A$.

It is shown in [3], that 0 satisfies (CEP), so we will of ten interchange the operator HS with $\mathrm{SH}$; and in the dual categories we will do likewise with GM and MG. Let $Z \in \operatorname{GM}(X)$, and let $e$ be an end of $Z$; define

$$
\sigma_{(z, e)}(x):=\prod_{\substack{0 \leq k \leq m-1 \\ 0 \leq l \leq m-1}}\left(g^{k}(x) \leq g^{2}(x) \mid g^{k}(e) \leq g^{2}(e) \text { in } z\right)
$$

and let

$$
\sigma(x):=W\left(\sigma_{(z, e)}(x) \mid z \in \operatorname{GM}(X) \text { and } e \text { is an end of } z\right) \text {. }
$$

THEOREM 2.11. $K=\{A \in 0 \mid P(A) \vDash(\forall x) \sigma(x)\}$.

Proof. Let $A \in 0$, and suppose $P(A) \models(\forall x) \sigma(x)$. If $u \in P(A)$, then $P(A)=\sigma(u)$; that is, there exists $Z_{u}$ in $\operatorname{GM}(X)$ with end $e_{u}$,

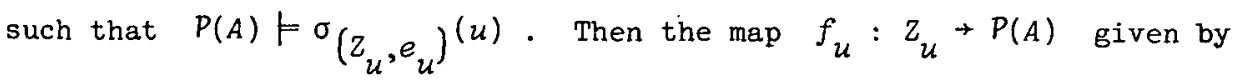
$f_{u}\left(g^{k}\left(e_{u}\right)\right)=g^{k}(u)$, is easily seen to be an S-morphism, with $\phi_{u}: A \rightarrow O\left(z_{u}\right)$, given by $a \phi_{u}=\left\{g^{k}\left(e_{u}\right) \mid g^{k}(u) \in a\right\}$, as its algebraic dual. Note that we are identifying $A$ with $O(P(A))$. Now if $a \neq b$ then without loss of generality there exists $u$ in $a-b$, so $e_{u}=g^{0}\left(e_{u}\right) \in a \phi_{u}-b \phi_{u} ;$ hence $a \phi_{u} \neq b \phi_{u} ;$ and the map

$$
\phi=\prod\left(\phi_{u} \mid u \in P(A)\right): A \rightarrow \prod\left(O\left(z_{u}\right) \mid u \in P(A)\right)
$$

is an embedding, whence $A \in \operatorname{ISP}(\operatorname{HS}(O(X)))=\mathrm{K}$.

Conversely, let $A \in \mathrm{K}$; since $O(X)$ is a subalgebra of $L_{m, n}$ for 
some $m>n, n \geq 0$, it follows that $A \in \operatorname{ISPS}\left(L_{m, n}\right)$. Let $u \in P(A)$ and consider the map $\phi_{u}: A \rightarrow L_{m, n}$ given by $a \phi_{u}=\left\{k \in \mathbb{Z}_{m} \mid g^{k}(u) \in a\right\}$. It is easily seen that $\phi_{u}$ is a homomorphism, with $h_{u}$, defined by $h_{u}(k)=g^{k}(u)$, as its dual map in $S$. Now $A \phi_{u} \in \mathrm{S}\left(I_{m, n}\right) \cap \mathrm{K}=\mathrm{Si}\left(\mathrm{P}_{\mathfrak{m}, \mathrm{n}}\right) \cap \mathrm{K}$ by Corollary 2.6. Therefore, $A \phi_{u} \in \operatorname{HS}(O(X))$. Dualizing this, we have $P\left(A \Phi_{u}\right) \in G M(X) ;$ and setting $Z_{u}=P\left(A \phi_{u}\right)$, with the end $e_{u}$ of $z_{u}$ being determined by 0 in $m_{n}$, and observing that $z_{u} \neq \sigma_{\left(z_{u}, e_{u}\right)}\left(e_{u}\right)$, and that $h_{u}$ embeds $z_{u}$ into $P(A)$, we obtain $P(A) \neq \sigma_{\left(z_{u}, e_{u}\right)}\left(h_{u}\left(e_{u}\right)\right)=\sigma_{\left(z_{u}, e_{u}\right)}(u)$. Hence $P(A) \vDash(\forall x) \sigma(x)$

From Corollary 2.6 we see that the subairectly irreducible algebras in $P_{\mathrm{m}, \mathbf{n}}$ are precisely the subalgebras of $L_{m, n}$. Again, let $\mathrm{K}=\operatorname{HSP}(O(X))$. A natural question to ask is: Under what conditions is it true that the subdirectly irreducibles in $K$ are precisely the subalgebras of $O(X)$ ? We now set out to answer this question; as will be seen in section 4 , the result has a lot of bearing on the discussion of injectivity in $K$.

A subdirectly irreducible algebra $A$ (in $K$ ) is called maximal if it has no proper extensions in $\mathrm{Si}(K)$. Remembering that $\mathrm{Si}(K)=\mathrm{HS}(O(X))$ (Proposition 2.5), and dualizing, we will say that $Z$ in $\operatorname{GM}(X)$ is maximal if it is not a 'strict' S-morphic image of any space in $G M(X)$; that is, if $y \in \mathbf{G M}(X)$ and $h: Y \rightarrow Z$ is onto, then $h$ is an isomorphism. Note that $X$ is maximal in $\operatorname{GM}(X)$. Our question will be answered in the affirmative when $X$ is the only maximal in $\operatorname{GM}(X)$. We begin with a result which is central to the ensuing discussion.

THEOREM 2.12. $R$ is a retract of $X$ if and only if $R$ is the loop.

Proof. Let $R$ be a retract of $X$. Then there is an injection $f: R \succ X$ and a surjection $h: X \rightarrow R$, such that $h f(r)=r$ for each $r$ in $R$. Now $f(R)$ is a $g$-subset of $X$, so $f\left(e_{R}\right)=g^{k}\left(e_{x}\right)$ for some $k$ in $\mathbb{Z}_{m}$. Since $h$ is onto, $h\left(e_{x}\right)=e_{R}$, hence 


$$
e_{R}=h f\left(e_{R}\right)=h g^{k}\left(e_{x}\right)=g^{k} h\left(e_{x}\right)=g^{k}\left(e_{R}\right) \text {, }
$$

that is, $R$ is the loop.

Conversely, let $e$ be the end of $X$ and define $h: X \rightarrow L$ by $h(e)=g^{k}(e)$, where $k$ is the unique natural number such that $i \leq k \leq m-1$ and $0 \equiv k \bmod (m-n)$. That $h$ is well-defined, onto, and commutes with $g$, is trivial. If $g^{l^{1}}(e) \leq g^{l_{2}}(e)$, then $g^{l_{1}+k}(e)$ is comparable with $g^{l_{2}+k}(e)$. If $k$ is even, then $h$ is order-preserving, and if $m-n$ is even, then $k \equiv 0 \bmod (m-n)$ implies $k$ is even. If $m-n$ and $k$ are both odd, then

$$
\begin{aligned}
g^{Z_{1}+k}(e) \geq g^{Z_{2}+k}(e) & \Rightarrow g^{Z_{1}+k+m-n}(e) \leq g^{Z_{2}+k+m-n}(e) \\
& \Rightarrow g^{Z_{1}+k}(e) \leq g^{Z^{+k}}(e) \\
& \Leftrightarrow g^{Z_{1}+k}(e)=g^{Z_{2}+k}(e) .
\end{aligned}
$$

Thus $h$ is order-preserving. Finally, if $i \geq n$, then

$$
h g^{i}(e)=g^{i+k}(e)=g^{i+z(m-n)}(e)=g^{i-n z} g^{m z}(e)=g^{i-n z} g^{n z}(e)=g^{i}(e) \text {. }
$$

Therefore $h$ is retraction.

Now let $Z$ be maximal in $G M(X)$. Since $M G=G M, Z$ is (isomorphic to) an S-morphic image of a $g$-subset of $X$; and since $Z$ is maximal, it is in fact (isomorphic to) a $g$-subset of $X$. Thus $Z \cong X_{k}$ for some $k \leq n$. If $n=0$, then $X$ is a loop and hence $O(X)$ is simple, and has only trivial homomorphic images. If $n=1$, then $X_{1}$ is the loop of $X$, and by Theorem 2.12, $X_{1}$ is indeed an S-morphic image of $X$ and so $Z \cong X$. Finally, if $n \geq 2$, an easy argument shows that for each $k \leq n-2, x_{k+2}$ is an S-morphic image of $x_{k}$ via $g^{2}$. Hence the only maximals are (isomorphic to) $X$ and $X_{1}$.

Now if $m=1$ then $X$ is the only object in $\operatorname{GM}(X) ;$ and if $m=2$, either $X$ is the loop or $X_{1}$ is the loop: see Figure 2. Thus for $m \leq 2$, the only maximal in $\operatorname{GM}(X)$ is $X$. 
9
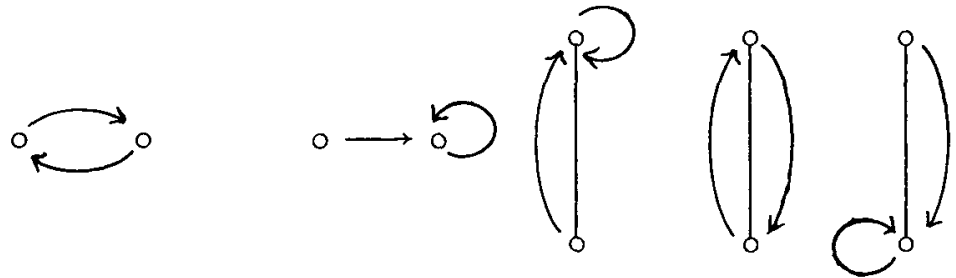

FIGURE 2. Duals of subdirectly irreducibles with $m \leq 2$

Furthermore, if $X$ is a 3-element chain, then $x_{1}$ is either the loop, in which case $X_{1}$ is not maximal in $G M(X)$, or the midale element of $X$ is a $g$-fixed point, in which case there can be no S-morphism from $X$ onto $X_{1}$, whence $X_{1}$ is maximal in $\operatorname{GM}(X)$. This situation is illustrated in Figure 3.
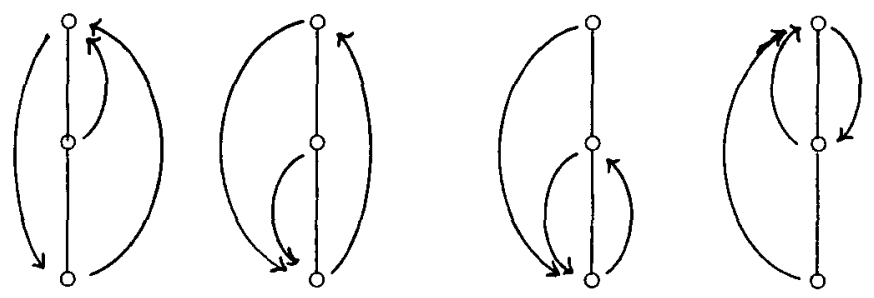

$X_{1}$ is not maximal
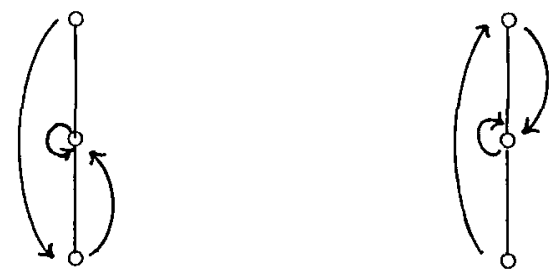

$X_{1}$ is maximal

FIGURE 3. Case where $X$ is a 3 -element chain 
LEMMA 2.13. If $m \geq 4$, and $X$ is a chain, then $X_{1}$ is maximal in $\operatorname{GM}(X)$.

Proof. It is easy to verify that $X_{I}$ cannot be the loop if $X$ is a chain with more than three elements. Now if there is an S-morphism $f$, from $X$ onto $X_{1}$, then $f\left(e_{X}\right)=e_{X_{1}}=g\left(e_{X}\right)$, but $f$ must then be orderpreserving and order-reversing, so, since we have $e_{X}$ comparable with $g\left(e_{X}\right)$, it follows that $g\left(e_{X}\right)=g^{2}\left(e_{X}\right)$. Thus $\operatorname{Card}(X)=2$, a contradiction.

In general when $m \geq 3$, the situation is more complicated, but we now have almost enough information to answer the question. The next result gives us a procedure for determining whether or not $X_{1}$ is maximal in $G M(X)$, merely by looking at a picture of $X$.

If $x, y$ are elements of a poset $P$ then we will write $x \not y$ if $x$ is comparable with $y$, and $x \| y$ if $x$ is incomparable with $y$.

THEOREM 2.14. Let $m \geq 3$, and suppose $X$ is not a 2oop. Then $x_{1}$ is maximal in $\operatorname{GM}(X)$ if and only if $X$ is a chain with more than three elements or the following four conditions hold:

(i) $X$ is not an antichain;

(ii) $X_{1}$ is not the loop;

(iii) if $k$ is the smallest natural number with $g^{k}(e)<g^{k+1}(e)$ or $g^{k}(e)>g^{k+1}(e)$, then $g^{k+1}(e) \neq g^{k+2}(e)$;

(iv) if for all natural numbers $k, g^{k}(e) \| g^{k+1}(e)$, then there are at least two distinct pairs of elements related by $<$.

Proof. Let $X_{1}$ be maximal in $G M(X)$, and suppose $X$ is not a chain. Conditions $(i)$ and $(i i)$ follow immediately from Corollary 2.6 and Theorem 2.12. 
(iii) Let $k$ be the smallest natural number with $g^{k}(e)<g^{k+1}(e)$ or $g^{k}(e)>g^{k+1}(e)$ (assuming such exists): note that $k \neq 0$ since $m \geq 3$. Suppose $g^{k+1}(e)=g^{k+2}(e)$. If $g^{k-1}(e) \# g^{k+1}(e)$, without loss of generality assume $g^{k-1}(e) \leq g^{k+1}(e)$, then $g^{k}(e) \geq g^{k+2}(e)=g^{k+1}(e)$. But then $g^{k-1}(e) \leq g^{k+1}(e) \leq g^{k}(e)$, a contradiction. Hence $g^{k-1}(e) \| g^{k+1}(e)$. Now let $0<s \leq r-1<k$, and suppose $g^{k-r}(e) \# g^{k-s}(e)$; then

$$
g^{k-1}(e)=g^{k-r+r-1}(e) \# g^{k-s+r-1}(e)=g^{k+r-(s+1)}(e)=g^{k+1}(e) \text {, }
$$

contradicting the fact that $g^{k-1}(e) \| g^{k+1}(e)$. Now define $f: X \rightarrow X_{1}$ by $f(e)=g(e)$. It is easily seen that $f$ is well-defined, onto, and commutes with $g$; and since the only order to preserve, namely $g^{k}(e) \# g^{k+1}(e)$, is preserved because we have assumed $g^{k+1}(e)=g^{k+2}(e)$, it follows that $X_{1}$ is an S-morphic image of $X$, contradicting the maximality of $X_{1}$.

(iv) Assume the hypothesis, and suppose $g^{r}(e)<g^{s}(e)$ ' is the only non-trivial order relationship holding in $x$. Now $g^{r+1}(e) \geq g^{s+1}(e)$, but if $g^{p+1}(e)>g^{s+1}(e)$, then $g^{r+1}(e)=g^{s+1}(e)$; but then $f: X \rightarrow X_{1}$ defined as in ( $i i i)$ is again order-preserving, contradicting the maximailty of $x_{1}$.

Suppose on the other hand, that $X_{1}$ is not maximal in $\operatorname{GM}(X)$; that is, there exists an S-morphism $f: X \rightarrow X_{1}$. Further, suppose $X_{1}$ is not the loop, and $X$ is not an antichain, so $f=g$. Now, either

(a) there exists a natural number $k$, such that $g^{k}(e) \# g^{k+1}(e)$, or

(b) $g^{k}(e) \| g^{k+1}(e)$ for each natural number $k$. If (a), then let $k_{0}$ be the smallest such number. Without loss of 
generality, suppose $g^{k_{0}}(e)<g^{k_{0}+1}(e)$; then $g^{k_{0}+1}(e) \geq g^{k_{0}+2}(e)$. But $f$ is order-preserving, and $f=g$, thus $g^{k_{0}+1}(e)=g^{k_{0}+2}(e)$. So (iii) fails, and $X$ also cannot be a chain. If (b), then let $r$ be the smallest natural number involved in a non-trivial order relationship; that is $g^{r}(e) \# g^{s}(e)$ and $g^{r}(e) \neq g^{s}(e)$ for some $s$ in $N$. Without loss of generality, assume $g^{r}(e)<g^{s}(e)$; then by the same argument as above, $g^{r+1}(e)=g^{s+1}(e)$. Suppose there is another pair of elements $(u, v)$ related by <; that is, $g^{u}(e)<g^{v}(e)$. Then we again have $g^{u+1}(e)=g^{v+1}(e)$. But $g^{v+1}(e)=g^{u+1}(e)=g^{r+1+u-r}(e)=g^{s+1+u-r}(e)=g^{u+1+s-r}(e)=g^{v+1+s-r}(e)$. Therefore, $s-r \equiv 0 \bmod (m-n) ;$ whence $g^{s}(e)=g^{r}(e)$, a contradiction. So (iv) fails.

It follows from this result and its proof that $X_{1}$ is non-maximal in $\operatorname{GM}(X)$ if and only if one of the following hold.

(0) $X$ is isomorphic to one of the spaces in Eigure 2;

(1) $X$ is an antichain;

(2) $X_{1}$ is the loop of $X$ (regardless of the order relations on $X$ );

(3) $X$ is isomorphic to the space (A) in Figure 4, or its dual;

(4) $X$ is isomorphic to the space (B) in Figure 4, or its dual.

(A)

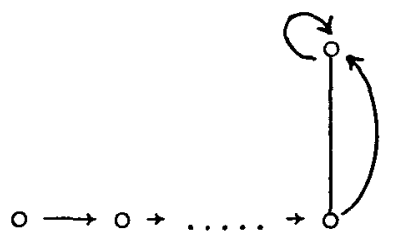

(B)

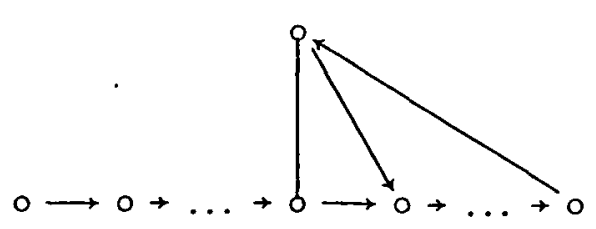

FIGURE 4

COROLLARY 2.15. Let $A$ be a finite set of finite subdirectly 
irreducible distributive Ockham algebras and let $\mathrm{K}=\mathrm{HSP}(\mathrm{A})$. Then the subdirecily irreducibles of $K$ are precisely all the subalgebras of algebras in $A$ if and only if for each $A$ in $A, P(A)$ satisfies one of the conditions (0)-(4) given above.

We close this section with a few remarks about the structure of $X$. Once again, the results obtained are indispensable to our discussion of injectivity in $\operatorname{Var}(O(X))$. First we consider the case when $X$ is a loop. If $m$ is even, define

$$
Y(m)=\left\{k \in \mathbb{Z}_{m} \mid k \text { odd, } 2 k \leq m\right\} .
$$

Let $Y \subseteq Y(m)$, and let $\leq_{Y}$ be the least reflexive relation on $X$, such that $e_{X} \leq_{Y} g^{k}\left(e_{X}\right)$ for each $k$ in $Y$, and $x_{1} \leq_{Y} x_{2}$ implies $g\left(x_{2}\right) \leq_{y} g\left(x_{1}\right)$ in $X$. A more general form of the following result is proved in [23].

LEMMA 2.16. (i) If $m$ is odd, then $X$ is an antichain.

(ii) If $m$ is even then the ordering on $X$ is isomorphic to some $\leq_{Y}$ where $Y \subseteq Y(m)$.

(iii) In case (ii) distinct subsets $y$ give rise to non-isomorphic Ockham spaces.

We note a few consequences of this result, remembering that in what follows, $m$ is even. Without loss of generality, we are choosing the end of $X$ to be a minimal element of $X$. If $g^{k}(e)<g^{l}(e)$, then $k$ is even and $l$ is odd, thus if $m$ is even and $Y=\varnothing$, then $X$ is an antichain, and if $Y \neq \varnothing$, then $X$ has height 1 . Finally, if $k \in Y$ then $e<g^{m-k}(e)$.

LEMMA 2.17. If $e<g^{k}(e)$, then $k \in Y$ or $m-k \in Y$.

Proof. Let $e<g^{k}(e)$, and suppose $k \notin Y$; then $2 k>m$. Also $g^{m-k}(e)>g^{m-k}\left(g^{k}(e)\right)=g^{m}(e)=e$ and since $m-k$ is odd, it follows from the second remark above that $e<g^{m-k}(e)$. Finally $2 k>m \Rightarrow 2 k-2 m>-m \Rightarrow 2(m-k)<m$. Hence $m-k \in Y$. 
Let $x, y$ be elements of a poset $P$; we write $x C y$ if $x$ and $y$ are connected in the graph of $P$, and $x \notin y$ if they are not.

THEOREM 2.18. If $X$ is not connected, then for each $k$ in $Y$, g.c.d. $(k, m) \neq 1$.

Proof. Let $k \in Y$, and suppose g.c.d. $(k, m)=1$. Then by [14, Corollary 6.4, p. 53], $g^{k}$ generates the cyclic group $\left\{g, g^{2}, \ldots, g^{m}\right\}$; that is, there exists $z$ in $\mathbb{Z}_{m}$ with $g^{z k}(e)=g(e)$. Now $k \in Y$, so $e<g^{k}(e)$. Thus

$$
g^{k}(e) \geq g^{2 k}(e) \leq g^{3 k}(e) \geq \ldots \# g^{(z-1) k}(e) \# g^{z k}(e)=g(e) .
$$

Therefore $e C g(e)$. But then $g^{2}(e) \mathrm{Cg}^{2-1}(e)$ for all $Z$ in $\mathbb{Z}_{m}$; that is $X$ is connected.

THEOREM 2.19. If $X$ is not connected, then each (connected) component of $X$ is self dual.

Proof. Suppose $X$ is not connected and $Y \neq \emptyset$. By Theorem 2.18, we can choose $k_{0}=\min (Y)$. We show that $P_{0}$, the component containing $e$, is self dual. Define $f: P_{0} \rightarrow P_{0}$ by $f\left(g^{2}(e)\right)=g^{m-Z-k_{0}}(e)$. If $g^{l}(e) \in P_{0}$, then since $e e^{m-k_{0}}(e)$, it follows that $g^{l}(e) \operatorname{Cg}^{m-k_{0}}(e)$. Thus $e=g^{m}(e)=g^{m-l+2}(e) c g^{m-l+m-k_{0}}(e)=g^{m-l-k_{0}}(e)$; that is, $f\left(g^{2}(e)\right) \in P_{0}$, hence $f$ is well-defined. If $g^{r}(e)<g^{s}(e)$, then $r$ is even and $s$ is odd, so $g^{m-k_{0}+r}(e) \geq g^{m-k_{0}+s}(e)$ from which it follows that $g^{m-k_{0}-s}(e) \leq g^{m-k_{0}-r}(e)$. Hence $f\left(g^{r}(e)\right) \geq f\left(g^{s}(e)\right)$, and $f$ is order-reversing. If $g^{r}(e) \in P_{0}$, then by the same argument as before, $g^{m-r-k_{0}}(e) \in P_{0}$, and $f\left(g^{m-r-k_{0}}(e)\right)=g^{m-m+r+k} 0(e)=g^{r}(e) ;$ showing that $f$ is onto. Finally, 


$$
\begin{aligned}
f\left(g^{r}(e)\right) \leq f\left(g^{s}(e)\right) & \Rightarrow g^{m-r-k} 0(e) \leq g^{m-s-k_{0}}(e) \\
& \Rightarrow g^{m-r}(e) \geq g^{m-s}(e) \\
& \Rightarrow g^{m+s}(e) \leq g^{m+r}(e) \\
& \Rightarrow g^{s}(e) \leq g^{r}(e)
\end{aligned}
$$

showing that $f$ is a dual order-embedding.

We now show that each component is dually isomorphic to the next. Since $X$ is not connected, it is clear that $g(e) \notin P_{0}$. Let $P_{I}$ be the component containing $g(e)$, and define $h: P_{0} \rightarrow P_{1}$ by $h\left(g^{2}(e)\right)=g^{2+1}(e)$; that is $h=g$ on $P_{0}$.

If $g^{2}(e) \in P_{0}$, then since $g$ preserves connectedness, it follows that $g^{l+1}(e) \in P_{l}$, thus $h$ is well-defined. By definition, $h$ is order-reversing; and if $g^{r}(e) \in P_{1}$, then $g^{r-1}(e)=g^{r+m-1}(e) C e \in P_{0}$ and $h\left(g^{r-1}(e)\right)=g^{r}(e)$, so $h$ is onto. Finally, if $h\left(g^{r}(e)\right) \leq h\left(g^{s}(e)\right)$ then $g^{r+1}(e) \leq g^{s+1}(e)$, and since $m-1$ is odd, we have $g^{r}(e)=g^{r+1+m-1}(e) \geq g^{s+1+m-1}(e)=g^{s}(e)$, thus $h$ is an order-embedding. Clearly this argument goes through for each pair of successive components. $\square$

We note in passing that the cases we are presently studying are by no means exhaustive; in fact the results obtained from Lemma 2.16 through to the end of Section 2, are basically no more than is necessary for the discussion of injectivity in Section 4.

Finally, we consider the case when $X$ is not a loop, and there exist $k, z$ in $\mathbb{Z}_{m}$, with $g^{k}(e)<e<g^{l}(e)$. Suppose $X$ is not connected. Let $s$ be the smallest natural number such that $g^{s}(e) \notin P_{0}$; that is $g^{s}(e) \$ e$. Since $g^{s}(e) \| g^{s-1}(e)$, it follows that $g^{s-1}(e) \| g^{s-i-1}(e)$ for each $i$ in $\{1, \ldots, s-1\}$. By induction, we see that $\left\{e, g(e), \ldots, g^{s-1}(e)\right\}$ forms an antichain. Now suppose $g^{r}(e) C e ;$ since $g^{2}(e)>e$, we have $g^{2}(e) C^{r}(e)$. But, by definition, $g^{s-1}(e) C e$, so 
$g^{s-1}(e) C g^{r}(e)$, and hence $g^{s}(e) C g^{r+1}(e) C g^{l+1}(e) \leq g(e) ;$ thus $s=1$. We have proved the following result.

LEMMA 2.20. If $X$ is not connected, then every element of each (connected) component is mapped to the next component by $g$.

If $X$ is not connected, let $p$ be the number of components; that is $x=P_{0}$ i $P_{1}$ i $\ldots$ u $P_{p-1}$.

LEMMA 2.21. Let $r \in \mathbb{Z}_{m}$; then $g^{r}(e) \in P_{0}$ if and only if $p$ divides $r$. More generally, $g^{r}(e) \in P_{i}$ if and only if $r \equiv i \bmod p$.

Proof. By Lemma 2.20, $g^{p}(e) \notin P_{p-1}$, and if $g^{p}(e) \in P_{1}$, then

$$
g^{p+1}(e) \in P_{2}, g^{p+2}(e) \in P_{3}, \ldots, g^{2 p-2}(e) \in P_{p-1} .
$$

But then $g^{2 p-1}(e) C g^{p}(e) \in P_{1}$, and no element is mapped into $P_{0}$ by $g$. A similar argument shows that $g^{p}(e) \notin P_{i}$ for each $i>0$. Thus $g^{p}(e) \in P_{0}$, and by applying $g$ repeatedly, we obtain $g^{z P}(e) \in P_{0}$, for each natural number $z$.

Conversely, let $g^{r}(e) \in P_{0}$, and suppose $r=z p+i$ where $1 \leq i \leq p-1$. Then, by Lemma 2.20 ,

$$
g^{r+1}(e) \in P_{1}, g^{r+2}(e) \in P_{2}, \ldots, g^{r+p-i}(e) \in P_{p-i} .
$$

But $g^{r+p-i}(e)=g^{z p+p+i-i}(e)=g^{(z+1) p}(e) \in P_{0}$. Thus $. p-i=0$, a contradiction.

\section{The free distributive 0ckham algebra generated by a distributive lattice}

For every object $X$ in $P$, define $s_{\omega}(X):=X^{\omega}$, and endow it with the product topology and the following order: for $x=\left(x_{i}\right)_{i \in \omega}$, $y=\left(y_{i}\right)_{i \in \omega}$ in $x^{\omega}$, 


$$
x \leq y \text { if and only if } x_{2 i+1} \geq y_{2 i+1} \text { and } x_{2 i} \leq y_{2 i} \text { in } x .
$$

That is, $s_{\omega}(x)=x \times x^{d} \times x \times x^{d} \times \ldots$, where $x^{d}$ denotes the order theoretic dual of $X$; we will call this the altemating order on $X^{\omega}$. Define $g$ on $s_{\omega}(X)$ by $g\left(\left(x_{i}\right)_{i \in \omega}\right)=\left(x_{i+1}\right)_{i \in \omega}$. For each map $f$ in $\mathbf{P}(X, Y)$, define $S_{\omega}(f): S_{\omega}(X) \rightarrow S_{\omega}(Y)$ by $S_{\omega}(f)\left(\left(x_{i}\right)_{i \in \omega}\right)=\left(f\left(x_{i}\right)\right)_{i \in \omega}$. This gives a functor from $P$ into $S$. For a finite subdirectly irreducible distributive Ockham algebra $A$, we obtain a functor from $S$ into $\mathrm{S}_{A}$, the dual category of $\operatorname{Var}(A)$, by setting

$$
S_{A}(X)=\{x \in X \mid X \vDash \sigma(x)\} \text {, for each } X \text { in } S .
$$

(Here $\sigma(x)$ is defined as for Theorem 2.11.) The topology, order, and the map $g$, are those induced on the subspace $S_{A}(X)$ by $X$. When $A=L_{m, n}$ for some $n \geq 0, m>n$, we will abbreviate $S_{A}=S_{L_{m, n}}$ to $S_{m, n}$.

THEOREM 3.1. (i) The functor $S_{\omega}: P \rightarrow S$ is right adjoint to the forgetful functor.

(ii) The functor $S_{A}: S \rightarrow S_{A}$ is right adjoint to the inclusion functor.

Before giving the proof, we observe that via Priestley's dualj.ty, this theorem gives us left adjoints to the forgetful functor from 0 into $D$, and the inclusion functor from $\operatorname{Var}(A)$ onto 0 . (By composing, we also obtain the left adjoint to the forgetful functor from $\operatorname{Var}(A)$ into $D$. In general, if $K$ is a full subcategory of $L$, then $H: L \rightarrow K$, defined by $H(B)=B / \theta$, where $\theta=\wedge(\Theta \in \operatorname{Con}(B) \mid B / \theta \in K)$, is left adjoint to the inclusion functor from $K$ into $L$. In the second part of the theorem, we have $L=0$ and $K$ is generated by a single finite algebra, and we have described the congruence $\Theta$ on $B$ in 0 , by describing the appropriate closed $g$-subset of $P(B)$.

This approach has been used before by Davey and Goldberg in [10], to describe the free distributive p-algebras. For a more general categorical discussion of these ideas, see the remarks following Theorems 2.2 and 2.3 of that paper. 
Now, by noting that the dual space of $F D(K)$, the free bounded distributive lattice on $k$ generators, is $2^{k}$, we obtain the following description of free algebras in the categories 0 and $\operatorname{Var}(A)$ for any finite subdirectly irreducible distributive Ockham algebra $A$.

COROLLARY 3.2. (i) $\quad F D(k) \cong 0\left(S_{\omega}\left(2^{k}\right)\right) \cong O\left(\left(2^{k}\right)^{\omega}\right)$.

(ii) If $\mathrm{K}=\operatorname{Var}(A)$, then $F K(K) \cong 0\left(S_{A}\left(S_{\omega}\left(2^{K}\right)\right)\right) \cong 0\left(S_{A}\left(\left(2^{K}\right)^{\omega}\right)\right)$.

The description of the free algebras in 0 was first given in [23]. We note in passing, that when $A=L_{m, n}$ for some $n>0, m \geq n, \sigma(x)$ is the equation $g^{m}(x)=g^{n}(x)$. So in particular,

$$
F P_{m, n}(\kappa) \cong O\left(\left\{x \in\left(2^{\kappa}\right)^{\omega} \mid g^{m}(x)=g^{n}(x)\right\}\right)
$$

Proof of Theorem 3.1. (i) To see that $S_{\omega}$ is a well-defined functor, we first note that for $X$ in $P, X^{\omega}$ is compact and totally order-disconnected; that is, $S_{\omega}(X) \in P$. In fact, $S_{\omega}(X) \in S$, since $g$ is order-reversing by definition, and for any sub-basic open set $u$ of $s_{\omega}(X), g^{+}(u)=X \times u$, which is open in $s_{\omega}(X)$; hence $g$ is continuous.

Now let $f \in P(X, Y)$. It is easily seen that $s_{\omega}(f)$ is well-defined and order-preserving, since $f$ is order-preserving. To see that $S_{\omega}(f)$ is continuous, let $u=U_{0} \times U_{1} \times \ldots$ be a basic open set in $s_{\omega}(Y)$. Then

$$
\begin{aligned}
S_{\omega}(f)^{\leftarrow}(u) & =\left\{\left(x_{i}\right)_{i \in \omega} \in S_{\omega}(x) \mid\left(f\left(x_{i}\right)\right)_{i \in \omega} \in u\right\} \\
& =S_{\omega}(x) \cap\left(f^{+}\left(U_{0}\right) \times f^{+}\left(U_{1}\right) \times \ldots\right),
\end{aligned}
$$

which is open in $S_{\omega}(X)$, since $f$ is continuous. That $S_{\omega}(f)$ commutes with $g$ is trivial. Thus $S_{\omega}(f)$ is an S-morphism.

Finally, to verify that $S_{\omega}$ is right adjoint to the forgetful functor, $|-|: S \rightarrow P$, we must show that for each $X$ in $P$, for every $Y$ in $S$, and for all $\alpha$ in $P(|Y|, X)$, there exists a unique $B$ in 
$S\left(Y, S_{\omega}(X)\right)$, such that the diagram in Figure 5 commutes.

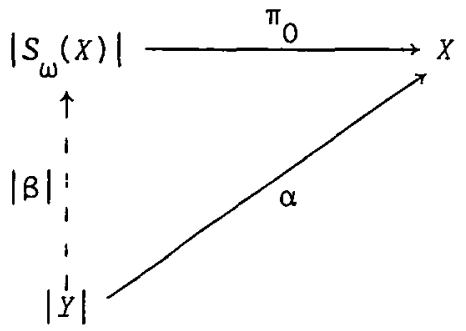

FIGURE 5

Let $\alpha \in P(|Y|, X)$, and define $\beta: Y \rightarrow S_{\omega}(X)$ by $\beta(y)=\left\langle\alpha g^{i}(y)\right\rangle_{i \in \omega}$. Clearly $B$ is well-defined and continuous, since $\alpha$ and $g$ are continuous. If $y \leq y^{\prime}$ in $Y$, then

$$
\pi_{2 i}(\beta(y))=\alpha g^{2 i}(y) \leq \alpha g^{2 i}\left(y^{\prime}\right)=\pi_{2 i}\left(\beta\left(y^{\prime}\right)\right)
$$

and

$$
\pi_{2 i+1}(\beta(y))=\alpha g^{2 i+1}(y) \geq \alpha g^{2 i+1}\left(y^{\prime}\right)=\pi_{2 i+1}\left(\beta\left(y^{\prime}\right)\right),
$$

so $\beta$ is order-preserving. And $\beta$ is an S-morphism since for all $y$ in $Y$,

$$
B(g(y))=\left\langle\alpha g^{i}(g(y))\right\rangle_{i \in \omega}=\left\langle\alpha g^{i+1}(y)\right\rangle_{i \in \omega}=g\left(\left\langle\alpha g^{i}(y)\right\rangle_{i \in \omega}\right)=g B(y) .
$$

Thus $\beta \in S\left(Y, S_{\omega}(X)\right)$. It is trivial that $\pi_{0}|\beta|=\alpha$. To see that $\beta$ is the unique map with this property, assume that there exists $\lambda$ in $S\left(Y, S_{\omega}(X)\right)$ with $\pi_{0}|\lambda|=\alpha$. Then, for each $y$ in $Y$,

$$
\begin{aligned}
B(y)=\left\langle\alpha g^{i}(y)\right\rangle_{i \in \omega}=\left\langle\pi_{0}|\lambda| g^{i}(y)\right\rangle_{i \in \omega} & =\left\langle\pi_{0} \lambda g^{k}(y)\right\rangle_{i \in \omega} \\
& =\left\langle\pi_{0} g^{i}(\lambda(y))\right\rangle_{i \in \omega}=\left\langle\pi_{i}((y))\right\rangle_{i \in \omega}=\lambda(y) .
\end{aligned}
$$

Thus $B=\lambda$.

(ii) To see that $S_{A}$ is a well-defined functor, we show that for $X$ in $\mathrm{S}, \mathrm{S}_{A}(X)$ is a closed $g$-subset of $X$. Let $x \in X-\mathrm{S}_{A}(X)$. Then 
$x \forall \sigma(x)$; that is $x \forall \sigma_{(Z, e)}(x)$ for all $Z$ in $\operatorname{GM}(P(A))$, and for each end $e$ of $Z$. Thus, for each $Z$ in $G M(P(A))$, and for every end $e$ of $Z$, there exist natural numbers $k$ and $l$, with $g^{k}(e) \leq g^{2}(e)$ but $g^{k}(x) \neq g^{2}(x)$ in $X$. By total order-disconnectedness, there exists a clopen order-ideal $U$ of $X$, with $g^{l}(x) \in U$ and $g^{k}(x) \notin U$. So $x \in\left(g^{2}\right)^{+}(U)-\left(g^{k}\right)^{+}(U)$, which is open in $X$, since $g$ is continuous and $U$ is clopen. Now if $y \in\left(g^{2}\right)^{+}(U)-\left(g^{k}\right)^{+}(U)$, then $g^{k}(y) \neq g^{2}(y)$, and clearly $X \not \neq \sigma(y)$; that is, $y \in X-S_{A}(X)$. Thus $S_{A}(X)$ is closed. We now show that $S_{A}(X)$ is g-invariant. Let $x \in S_{A}(X)$; then there exists $z$ in $G M(X)$, with end $e$, such that $g^{k}(e) \leq g^{l}(e)$ in $Z$ implies that $g^{k}(x) \leq g^{Z}(x)$ in $X$. If $O(Z)$ is simple, then by the remark following Corollary 2.2, we can choose any element of $Z$ to be an end. Let $e^{\prime}=g(e)$; then $g^{k}\left(e^{\prime}\right) \leq g^{l}\left(e^{\prime}\right)$ in $z$ implies that $g^{k+1}(e) \leq g^{l+1}(e)$ in $Z$, which implies that $g^{k+1}(x) \leq g^{2+1}(x)$ in $X$. Hence $X \vDash \sigma_{\left(z, e^{\prime}\right)}(g(x))$. If $O(Z)$ is not simple, then $W=z_{1}$ is a $g$-subset of 2 , and

$$
\begin{aligned}
g^{k}\left(e_{W}\right) \leq g^{l}\left(e_{W}\right) \text { in } W & \Leftrightarrow g^{k}(g(e)) \leq g^{l}(g(e)) \\
& \Rightarrow g^{k+1}(e) \leq g^{2+1}(e) \\
& \Rightarrow g^{k+1}(x) \leq g^{2+1}(x) \text { in } X \\
& \Rightarrow g^{k}(g(x)) \leq g^{2}(g(x)) \text { in } X .
\end{aligned}
$$

Hence $x \models \sigma_{\left(W, e_{W}\right)}(g(x))$. In either case $X \vDash \sigma(g(x))$; that is, $g(x) \in S_{A}(X)$. So $S_{A}(X)$ is a closed $g$-subset of $X$, and therefore $S_{A}(X) \in S$, by Theorem 1.1. That $S_{A}(X) \in S_{A}$ follows from the definition of $S_{A}(X)$ and Theorem 2.11, and a similar procedure to that used before, completes the proof.

We can actually obtain more explicit descriptions of free algebras in $P_{m, n}$, than those given above, but first we deviate slightly to look at coproducts, and not surprisingly, for distributive Ockham algebras, we 
obtain a similar result to the one derived by Berman and Dwinger [ 1 , Theorem 2, p. 216] for De Morgan algebras. The simple proof of that theorem used by Cornish and Fowler [9, Theorem 2.4], also. works here.

The product of a family of objects $\left(X_{i} \mid i \in I\right)$ in a category $x$ will be denoted by $\prod_{X}\left(x_{i} \mid i \in I\right)$; its coproduct will be denoted by $\perp_{X}\left(X_{i} \mid i \in I\right)$. If $X$ and $Y$ are two objects of $X$, then $X \prod_{X} Y$ and $X \perp_{X} Y$ will respectively denote the product and coproduct of ${ }^{*} X$ and $Y$ in $X$.

THEOREM 3.3. If $\left(x_{i} \mid i \in I\right)$ is a family of S-objects, then $\prod_{p}\left(X_{i} \mid i \in I\right)$ is an Ockham space and is the product of $\left(X_{i} \mid i \in I\right)$ in $\mathrm{S}$.

Proof. Define $g$ on $\prod_{P}\left(x_{i} \mid i \in I\right)$ by $g\left(\left\langle x_{i}\right\rangle_{i \in I}\right)=\left\langle g\left(x_{i}\right)\right\rangle_{i \in I}$. The proof is immediate from the fact that $g$ thus defined is continuous since each of its components is continuous.

Using Priestley's duality, the theorem gives us coproducts in $\mathbf{0}$; namely, for a family $\left(A_{i} \mid i \in I\right)$ of distributive Ockham algebras, $\perp_{0}\left(A_{i} \mid i \in I\right)$ is isomorphic as a lattice with $\perp_{D}\left(A_{i} \mid i \in I\right)$.

Consider again the varieties $K$ and $L$ with $K \subseteq L$, and let $H: L \rightarrow K$ be the left adjoint to the inclusion functor from $K$ into $L$. Since left adjoints preserve coproducts, $H\left(B \perp_{L} C\right)=H(B) \perp_{K} H(C)$, for all $B, C$ in $L$. But, if $K$ is a full reflective subcategory of $\mathrm{L}$, then $H(B) \cong B$ for all $B$ in $\mathrm{K}$; thus $B \perp \perp_{K} C \cong H\left(B \perp \perp_{L} C\right)$. Applying this to the case where $L=0$ and $K$ is generated by a single finite distributive Ockham algebra $A$, we have the following result.

THEOREM 3.4. If $\left(x_{i} \mid i \in I\right)$ is a famizy of $S_{A}$-objects, then

$$
\prod_{S_{A}}\left(x_{i} \mid i \in I\right) \cong S_{A}\left(\prod_{S}\left(x_{i} \mid i \in I\right)\right) ;
$$

that is,

$\prod_{S_{A}}\left(x_{i} \mid i \in I\right) \cong\left\{x \in \prod_{P}\left(x_{i} \mid i \in I\right) \mid \prod_{P}\left(x_{i} \mid i \in I\right) \vDash \sigma(x)\right\}$. 
Again, via Priestley's duality, we obtain coproducts in $\operatorname{Var}(A)$. If $A=L_{m, n}$, then for each $i$ in $I, g^{m}(x)=g^{n}(x)$ for all $x$ in $X_{i}$. Thus $g^{m}(x)=g^{n}(x)$ for all $x$ in $\prod_{p}\left(x_{i} \mid i \in I\right)$. Hence we have the following useful corollary.

COROLLARY 3.5. For $n \geq 0, m>n$, if $\left(B_{i} \mid i \in I\right)$ is a famizy of algebras in $\mathrm{P}_{\mathrm{m}, \mathrm{n}}$, then $\perp_{\mathrm{P}_{\mathrm{m}, \mathrm{n}}}\left(B_{i} \mid i \in I\right)$ and $\perp_{\mathbb{D}}\left(B_{i} \mid i \in I\right)$ are isomorphic lattices.

Observe that the class of algebras in $P_{2,0}$ which satisfy the identities $x \wedge \sim x=0$ and $x \vee \sim x=1$, is the class of Boolean algebras, denoted by B. The following result extends Corollary 2.6 of [9].

COROLLARY 3.6. If $\left\{B_{i} \mid i \in I\right\}$ is a family of Boolean algebras, then

$$
\begin{aligned}
& \perp_{\mathrm{B}}\left(B_{i} \mid i \in I\right) \cong \perp_{P_{2,0}}\left(B_{i} \mid i \in I\right) \cong \perp_{\mathrm{P}_{\mathrm{m}, \mathrm{n}}}\left(B_{i} \mid i \in I\right) \\
& \cong \perp_{0}\left(B_{i} \mid i \in I\right) \cong \perp_{\mathrm{D}}\left(B_{i} \mid i \in I\right) .
\end{aligned}
$$

Let $X$ be an ordered topological space and let $L$ be a bounded distributive lattice endowed with the discrete topology. Then $C_{\leq}(X, L)$ will denote the set of all continuous order-preserving functions from $X$ into $L$, and $\bar{C}_{\leq}(X, L)$, the subset of $C_{\leq}(X, L)$ consisting of those functions whose range is finite. It is easily seen that $C_{\leq}(X, L)$ is a bounded distributive lattice under the pointwise operations. The following result extends Theorem 2.7 of [9], to which the reader is referred for the proof.

THEOREM 3.7. Let $X$ be an ordered topological space with a continuous order-reversing map $g$ on it. Let $L$ be a distributive Ockham algebra. Then $\bar{C}_{\leq}(X, L)$ is a distributive ockhom algebra, where, for each $f$ in $\bar{C}_{\leq}(X, L), \sim f$ is defined by $(\sim f)(x)=\sim(f(g(x)))$ for each $x$ in $X$. Moreover, $\bar{C}_{\leq}(X, L)$ is isomorphic to $O(X) \perp_{0} L$.

We now return to free algebras. Berman [3], in generalizing De Morgan algebras, restricted his attention to the subvarieties $P_{2 m+n, n}$; he 
called these classes $K_{m, n}$. Urquhart [23], correctly asserted that many of the results actually go through for all of the $P_{m, n}$. However, when we study free algebras in $P_{m, n}$, it becomes apparent that the relationship between $m$ and $n$ - for example, whether they differ by an even number or not - is indeed significant.

Let $m>n, n \geq 0$, and let $x \in \mathrm{P}$. If $m-n$ is even, then $x_{n}^{m}$, the product space $x^{m}$ with the alternating order, and $g$ defined by

$$
g\left(\left(x_{0}, \ldots, x_{m-1}\right\rangle\right)=\left\langle x_{1}, \ldots, x_{m-1}, x_{n}\right\rangle,
$$

is easily seen to be an Ockham space. In fact, it is an object of $S_{m, n}$, the dual category of $P_{m, n}$.

THEOREM 3.8. If $n \geq 0, m>n$, and $m-n$ is even, then for each $x$ in $\mathrm{P}, \mathrm{S}_{m, n}\left(x^{\omega}\right)=x_{n}^{m}$.

Proof. Define $\eta: S_{m, n}\left(x^{\omega}\right) \rightarrow x_{n}^{m}$ by $\eta\left(\left\langle x_{0}, \ldots\right\rangle\right)=\left\langle x_{0}, \ldots, x_{m-1}\right\rangle$. It is easy to check that $\eta$ is well-defined, onto, order-preserving, open and continuous. To see that $\eta$ commutes with $g$, note that for each $x$ in $S_{m, n}\left(x^{\omega}\right), g^{m}(x)=g^{n}(x)$; thus $\pi_{m}(x)=\pi_{n}(x)$. So

$$
\begin{aligned}
& \left.n\left(g\left(x_{0}, \ldots, x_{m-1}, x_{n}, \ldots\right\rangle\right)\right)=n\left(\left(x_{1}, \ldots, x_{m-1}, x_{n}, \ldots\right\rangle\right) \\
& \quad=\left(x_{1}, \ldots, x_{m-1}, x_{n}\right)=g\left(\left\langle x_{0}, \ldots, x_{m-1}\right\rangle\right)=g n\left(\left\langle x_{0}, \ldots, x_{m-1}, x_{n}\right\rangle\right) .
\end{aligned}
$$

Finally, assume that $n\left(\left\langle x_{0}, \ldots\right\rangle\right)=n\left(\left\langle y_{0}, \ldots\right\rangle\right)$; that is, $\left\langle x_{0}, \ldots, x_{m-1}\right\rangle=\left\langle y_{0}, \ldots, y_{m-1}\right\rangle$. Again using the fact that $g^{m}(x)=g^{n}(x)$, we have for $k \geq 0, g^{m+k}(x)=g^{n+k}(x)$. Thus, by induction, $\pi_{m+k}(x)=\pi_{n+k}(x)=\pi_{n+k}(y)=\pi_{m+k}(y)$. Hence $n$ is one-toone, and similarly, $\eta$ is an order-isomorphism.

Now in general, if $x^{m}$ is given the alternating order, there is not always an Ockham space structure on $x^{m}$ such that $s_{m, n}\left(x^{\omega}\right) \cong X^{m}$. We note however, that there is a one-to-one correspondence between the 
elements $x=\left(x_{0}, \ldots\right\rangle$ of $s_{m, n}\left(x^{\omega}\right)$, and the $m$-tuples $\left(x_{0}, \ldots, x_{m-1}\right)$ consisting of the first $m$ components of $x$. Thus $\operatorname{Card}\left(S_{m, n}\left(X^{\omega}\right)\right)=\operatorname{Card}\left(X^{m}\right)=(\operatorname{Card}(X))^{m}$, and in fact $S_{m, n}\left(X^{\omega}\right)$ and $x^{m}$ are isomorphic in $P$.

If $m$ is odd, and $x, y \in S_{m, 0}\left(x^{\omega}\right)$ with $x \leq y$, then since $g$ is order-reversing, we have $x=g^{m}(x) \geq g^{m}(y)=y$. Thus $s_{m, 0}\left(x^{\omega}\right)$ is a Boolean space.

Finally, we look at the case $n=m-1$. Observe that for $x, y$ in $S_{m, m-1}\left(x^{\omega}\right)$, if $x \leq y$ then $\pi_{m-1}(x)=\pi_{m-1}(y)$. Now for each $y$ in $X$, the product space $x_{y}^{m-1}=\left\{\left(x_{0}, \ldots, x_{m-2}\right\rangle \mid x_{i} \in X\right\}$ with the alternating order and $g_{y}: x^{m-1} \rightarrow x^{m-1}$ defined by

$$
g_{y}\left(\left\langle x_{0}, \ldots, x_{m-2}\right)\right)=\left\langle x_{1}, \ldots, x_{m-2}, y\right\rangle
$$

is an Ockham space, and is in fact in $S_{m, m-1}$.

THEOREM 3.9. Let $m \geq 1$, and let $X \in P$. If $X$ is finite, then $S_{m, m-1}\left(x^{(\omega)}\right) \cong \dot{U}\left(x_{y}^{m-1} \mid y \in X\right)$.

Proof. Define $n: S_{m, m-1}\left(x^{\omega}\right) \rightarrow \dot{U}\left(x_{y}^{m-1} \mid y \in X\right)$ by $\eta\left(\left\langle x_{0}, \ldots, x_{m-2}, x_{m-1}, \ldots\right\rangle\right)=\left(x_{0}, \ldots, x_{m-2}\right\rangle$ in the component $x_{x_{m-1}}^{m-1}$.

It is easy to check that $\eta$ is well-defined, onto, ont-to-one, and continuous. By our earlier remark, $x \leq y$ implies that $n(x)$ and $n(y)$ are in the same component of the disjoint union, and hence $\eta$ is easily seen to be an order-isomorphism. Furthermore, $\eta$ commutes with $g$, since

$$
\begin{aligned}
r_{1} g\left(\left(x_{0}, \ldots\right)\right)=n\left(\left(x_{1}, \ldots\right)\right)=\left(x_{1}, \ldots, x_{m-2}, x_{m-1}\right\rangle \\
=g_{x_{m-1}}\left(\left\langle x_{0}, \ldots, x_{m-2}\right\rangle\right)=g_{x_{m-1}} n\left(\left\langle x_{0}, \ldots\right\rangle\right) .
\end{aligned}
$$

Now, translating all of the above results to the algebraic categories 
and recalling that in any variety $K$, $F K(K)$ is a coproduct in $K$ of $K$ copies of $F K(1)$, we can use Corollary 3.5 to obtain the following descriptions of free algebras in the various subvarieties.

COROLLARY 3.10. (i) For $m>n, n \geq 0, \mathrm{FP}_{\mathrm{m}, \mathrm{n}}(\mathrm{k})$ and $F D(\mathrm{~km})$ are isomorphic in $D$.

(ii) For $m-n$ even, $\mathrm{FP}_{\mathrm{m}, \mathrm{n}}(\mathrm{K})$ and $\mathrm{FD}(\mathrm{km})$ are isomorphic in $\mathrm{P}_{\mathrm{m}, \mathrm{n}}$

(iii) For $m>0, m$ odd, $\mathrm{FP}_{\mathrm{m}, 0}(\mathrm{k}),[\mathrm{FD}(0)]^{2^{m k}}$ and $2^{2^{m \kappa}}$ are isomorphic in $\mathrm{P}_{\mathrm{m}, 0}$.

(iv) For $m \geq 1, F P_{m, m-1}(\kappa)$ and $[F D(\kappa(m-1))]^{2}$ are isomorphic in $\mathrm{P}_{\mathrm{m}, \mathrm{m}-1}$.

Part (i) was first proved for De Morgan algebras by Berman and Dwinger [see 1, Theorem 3, p. 218].

\section{Injective distributive Ockham algebras}

Let $K$ be a class of algebras. An algebra $I$ in $K$ is (weak) injective if for each algebra $A$ in $K$, and every subalgebra $B$ of $A$, every homomorphism (epimorphism) $\phi: B \rightarrow I$ extends to a homomorphism $\bar{\phi}: A \rightarrow I$ with $\left.\bar{\phi}\right|_{B}=\phi ; I$ is self-injective if it is injective in the class $\{I\}$, that is, every homomorphism from a subalgebra of $I$ into $I$ extends to an endomorphism of $I$; and $I$ is an absolute subretract in $K$ if it is a retract of each of its extensions in $K$. We say that $K$ has enough injectives if every algebra in $K$ has an injective extension in $K$. We recall here the dual concept projectivity. Let $X \subseteq S$; an object $P$ in $X$ is (weak) projective, if for each object $X$ in $X$, and every surjection $h: X \rightarrow Y$ for some object $Y$ in $X$, every (mono)morphism $f: P \rightarrow Y$ lifts to a morphism $\bar{f}: P \rightarrow X$ with $h \circ \bar{f}=f$. We have already noted that injections in 0 correspond with surjections in $S$; thus an algebra $A$ is injective in 0 if and only if $P(A)$ is projective in $S$; this fact will be used in most of the ensuing proofs. A class $K$ of algebras is said to satisfy the amalgamation property if 
(AP): for all algebras $A, B_{i}(i=1,2)$ in $K$, and injections $\phi_{i}: A \rightarrow B_{i}(i=1,2)$, there exists an algebra $C$ in $\mathrm{K}$ and injections $\psi_{i}: B_{i} \rightarrow C$ $(i=1,2)$ such that $\phi_{1} \psi_{1}=\phi_{2} \psi_{2}$.

The following is a summary of the results we require about injectivity. The proofs can be found in [12] and [16].

PROPOSITION 4.1. Let $\mathrm{K}$ be a variety.

(i) Direct products of injective algebras in $\mathrm{K}$ are injective in K.

(ii) Regracts of injective algebras in $K$ are injective in $K$.

(iii) Every injective algebra in $\mathrm{K}$ is a retract of each of its extensions in $K$.

(iv) If $K$ has enough injectives then $K$ satisfies (AP) and (CEP).

(v) Let $A$ be a finite set of finite algebras and assume that $K:=\operatorname{ISP}(A)$ is congruence distributive. Let $A \in A$ and suppose that every subalgebra of $A$ is either subdirectly irreducible or weak injective in $\mathrm{K}$; then $A$ is injective in $\mathrm{K}$ if and only if it is injective in A.

(vi) Let $A$ be a finite algebra all of whose subalgebras are either subdirectly irreducible or weak injective in $K$, and assume that $\mathrm{K}:=\operatorname{ISP}(A)$ is congmence distributive. Then the following are equivalent:

(a) $A$ is injective in $\mathrm{K}$;

(b) $K$ has enough injectives;

(c) A is self-injective;

(d) $K$ satisfies $(\mathrm{AP})$ and (CEP).

Let $X$ be an Ockham space. Each $x$ in $X$ determines a map $f_{x}: N \rightarrow X$, defined by $f_{x}(k)=g^{k}(x)$. Let $\bar{f}_{x}: \beta N+X$ be the unique extension of $f_{x}$. Since $f_{x}$ commutes with $g$ on $N$, it follows that $\bar{f}_{x}$ commutes with $g$ on $B N$. Recall from the proof of Theorem 2.11, that 
for $X$ in $S_{A}$, the dual of the variety generated by a finite algebra $A$, each $x$ in $X$ determines a space $Z_{x}$ in $G M(P(A))$, an end $e$ of $Z_{x}$, and a similar map $f_{x}: Z_{x} \rightarrow X$. These maps correspond respectively to $\bar{\phi}_{x}: O(X)+L_{\omega}$ given by $U \bar{\Phi}_{x}=\left\{\overline{k \in N \mid g^{k}(x) \in U}\right\}$ and $\phi_{x}: O(X) \rightarrow O\left(z_{x}\right)$ given by $U \phi_{x}=\left\{g^{k}(e) \in z_{x} \mid g^{k}(x) \in U\right\}$. The following results are quite useful.

LEMMA 4.2. (i) Let $X \in S$, and let $x, y \in X$ with $x \neq y$. Then $\bar{f}_{x} \neq \bar{f}_{y}$

(ii) Let $X \in \mathrm{S}_{A}$, and let $x, y \in X$ with $x \neq y$. Then $f_{x} \neq f_{y}$. Proof. Suppose $\bar{f}_{x}=\bar{f}_{y}$. Then

$$
x=g^{0}(x)=\bar{f}_{x}(0)=\bar{f}_{y}(0)=g^{0}(y)=y \text {. }
$$

The proof of (ii) is similar and is in fact given in the proof of Theorem 2.11.

LEMMA 4.3. (i) Let $X \in S$. Every S-morphism from $\beta N$ into $X$ is of the form $\bar{f}_{x}$ for some $x$ in $x$, and $\bar{f}_{x}$ is uniquely determined by its value at 0 .

(ii) Let $X \in \mathrm{S}_{A}$. Every S-morphism from a g-subset $Z$ (with end $e$ ) of an S-morphic image of $P(A)$ into $X$ is of the form $f_{x}$ for some $x$ in $X$, and $f_{x}$ is uniquely determined by its value at $e$.

Proof. Again we only prove (i) since the proof of (ii) is almost identical. Let $f, f^{\prime} \in S(B N, X)$ and suppose $f(0)=f^{\prime}(0)$. Let $k \in N$; if $f(k)=f^{\prime}(k)$, then

$$
f(k+1)=f(g(k))=g f(k)=g f^{\prime}(k)=f^{\prime}(g(k))=f^{\prime}(k+1) .
$$

So, by induction, $f=f^{\prime}$ on $\mathrm{N}$, and hence $f=f^{\prime}$ on BN. Thus if $f$ and $f^{\prime}$ are distinct, then they take different values at 0 .

It follows that if $A \in 0$, then every prime ideal of $A$ determines a homomorphism of $A$ into $L_{\omega}$, and conversely, every homomorphism of $A$ 
into $L_{\omega}$ is determined by a prime ideal of $A$. This extends the corresponding result for De Morgan algebras proved in [4].

THEOREM 4.4. $L_{\omega}$ is an injective distributive ockham algebra.

Proof. We will show that $B N$ is projective in $S$. Let $X, Y \in S$ and let $h$ be an S-morphism from $Y$ onto $X$. Let $\bar{f}_{x} \in \mathrm{S}(B N, X)$ for some $x$ in $X$. Now, since $h$ is onto, the set $h^{+}(\{x\})$ is non-empty. Let $y \in h^{*}(\{x\})$; then $\bar{f}_{y}:$ BN $\rightarrow y$ lifts $\bar{f}_{x}$, because for each $n$ in $N$,

$$
\bar{f}_{x}(n)=f_{x}(n)=g^{n}(x)=g^{n} h(y)=h\left(g^{n}(y)\right)=h f_{y}(n)=h \bar{f}_{y}(n) .
$$

Since $0=\operatorname{ISP}\left(L_{\omega}\right)$, Proposition 4.1 (i) tells us that 0 has enough injectives. So applying Proposition 4.1 (iv), we have:

COROLLARY 4.5. 0 satisfies (AP) and (CEP).

We would obviously like to characterize all of the injectives in 0 , but unfortunately, we are as yet unable to do so, and leave this as an open problem. The following results are useful steps towards its solution, and collect together all of the information that is known to us at present.

LEMMA 4.6. Any injective distributive Ockham algebra is a complete Boolean lattice.

Proof. By Theorem 3.1, $O\left(s_{\omega}\right)$ is left adjoint to the forgetful functor $|-|$, from 0 into $D$; and since $s_{\omega}$ preserves onto maps, $O\left(S_{\omega}\right)$ preserves embeddings. Hence by a result of Banaschewski [2, p. 136], $|-|$ preserves injectivity. Thus if $A$ is injective in 0 , then $|A|$ is injective in $D$. But we know the injectives in $D$; they are the complete Boolean algebras (see for example [16, Theorem 14, p. 146]). Therefore $|A|$ is a complete Boolean lattice.

In fact, if $A$ is injective in 0 , we can actually say more. Recall that $0=\operatorname{ISP}\left(L_{\omega}\right) ;$ in fact $A$ is embedded into $L_{\omega}^{P(A)}$, and hence the identity map on $A$ extends to an epimorphism from $L_{\omega}^{P(A)}$ into $A$. Thus 
$A$ is actually a retract of $L_{\omega}^{P(A)}$.

LEMMA 4.7. If $A$ is injective in 0 , then for each $n \geq 1, P(A)$ has no $g^{n}$-fixed points.

Proof. Suppose that $y$ in $P(A)$ is a $g^{n}$-fixed point for some $n \geq 1$. Let 1 be the one-element Ockham space, and consider $I$ as an S-morphic image of $n+1_{0}$. Now $f: P(A) \rightarrow 1$ defined by $f(x)=1$ for each $x$ in $P(A)$ is an S-morphism, and since $P(A)$ is projective, there is an S-morphism $\vec{f}: P(A) \rightarrow n+1_{0}$, lifting $f$. But then

$g^{n} \bar{f}(y)=\bar{f} g^{n}(y)=\bar{f}(y)$, which is impossible in $n+1_{0}$.

By the above result, there are no finite injective distributive Ockham algebras.

We now turn our attention to the subvarieties of 0 which are generated by a single finite algebra. Our work is somewhat simplified due to the results of Davey and Werner [11]. We recall the following definitions from there. For an algebra $A$ and a Boolean algebra $B$, the bounded Boolean power $A[B]$ is defined to be the algebra $C\left(X_{B}, A\right)$ of continuous functions from the Boolean space $X_{B}$ of prime ideals of $B$, into $A$, where $A$ is endowed with the discrete topology. A simplicity formula for a class $K$ of algebras is an $\exists \forall$ conjunct of equations, say

$$
\alpha(a, b)=\operatorname{Ix} \forall y\left[\prod_{i \leq n} p_{i}(x, y, a, b)=q_{i}(x, y, a, b)\right],
$$

such that for each $A$ in $\mathrm{K}$,

$$
\{\theta(a, b) \in \operatorname{Con}(A)|A| \alpha(a, b)\}=\{\omega, \iota\} .
$$

Let $L$ be a subclass of $K$; if the (weak) injectives in $K$ are precisely the algebras of the form $\prod\left(A_{j}\left[B_{j}\right] \mid j \leq n\right)$ where the $A_{j}$ are pairwise nonisomorphic members of $\mathrm{L}$, and the $B_{j}$ are complete, then we say that the (weak) injectives in $K$ are induced by $L$.

THEOREM 4.8 ([11]). Let $K$ be a congruence-distributive variety generated by finitely many finite algebras, and assume that there is a simplicity formula for the maximal subdirectly irreducible algebras in $K$. 
Then the injectives (weak injectives) in $\mathrm{K}$ are induced by the subdirectly irreducibles which are injective in $\mathrm{K}$ (weak injective in $\mathrm{Si}(\mathrm{K})$ ).

We shall see anon that for each $m>n, n \geq 0, P_{m, n}$ has enough injectives. If $A$ is any finite subdirectly irreducible distributive Ockham algebra, we would like to know when $\operatorname{Var}(A)$ has enough injectives. This is not always the case. Recall that a De Morgan algebra which satisfies the identity $a \wedge \sim a \leq b \vee \sim b$, is called a Kleene algebra.

Cignoli [7] characterized the injective Kleene algebras; they are precisely the bounded Boolean powers of 3 - the three element Kleene algebra $\{0, a, 1\}$, with $\cdot 0=1, \sim 1=0,-a=a$. Now if $X$ is the Ockham space shown in Figure 6 , then the diagram cannot be completed, and hence $X$ is not projective in $\operatorname{GM}(X)$.

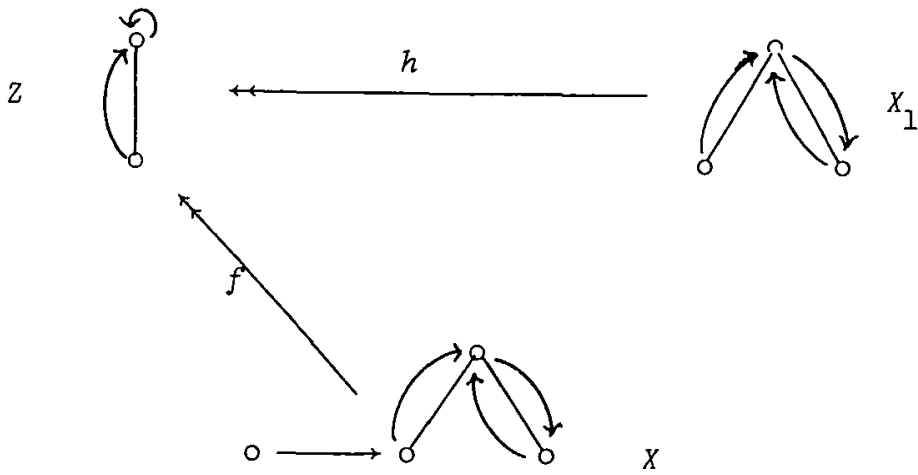

FIGURE 6

In fact, if $K=\operatorname{HSP}(O(X))$, then it is quite easily seen that $A$ is injective in $K$ if and only if $A$ is a Kleene algebra and injective as a Kleene algebra; that is, $A \cong 3[B]$, where $B$ is complete.

For the rest of this section, $X$ will be the dual of a finite subdirectly irreducible distributive Ockham algebra with end $e$, with $\operatorname{Card}(X)=m$, and $n$ the smallest number in $\mathbb{Z}_{m}$ such that $g^{m}(e)=g^{n}(e)$. We wish to characterize injectivity in $\operatorname{Var}(O(X))$. Our procedure will be to characterize projectivity in $S_{O}(X)$; the first step being to find the projectives in $G M(X)$. 
LEMMA 4.9. Let $2 \in \mathrm{GM}(X)$ and suppose $Z$ is a totally unordered 2oop. If $L$ is the loop of $X$ and $h$ in $S(L, Z)$ is onto, then any $f$ in $S(L, Z)$ lifts to a map $\bar{f}$ in $S(L, L)$.

Proof. If $\operatorname{Card}(Z)=1$, then the identity map on $L$ provides the lifting. Assume $\operatorname{Card}(Z)>1$; since $h$ is onto, it is clear that $L$ is not connected, and the number of (connected) components in $L$ equals Card( $Z$. If $L$ is itself an antichain, then $L$ is isomorphic to $Z$ and we are done. Otherwise, choose an end $e_{L}$ of $L$, and let $P_{0}$ be the component containing $e_{L}$. If $P_{i}=h^{\leftarrow}\left(f\left(e_{L}\right)\right)$, then define $\bar{f}: L \rightarrow L$ by $\bar{f}\left(e_{L}\right)=q$, where $q$ is the image of $e_{L}$ under the isomorphism from $P_{0}$ onto $P_{i}$ (see Theorem 2.19). It is easily verified that $\bar{f}$ is an S-morphism, and by its construction, $\bar{f}$ is also a lifting.

THEOREM 4.10. $L$ is projective in $\operatorname{GM}(X)$.

Proof. Let $Z \in G M(X)$. If $f \in S(L, Z)$, then $Z$ is a loop, and it is sufficient to show that $L$ is self-projective. Let $h$ in $S(L, Z)$ be onto, let $e_{L}$ be the end of $L$, and define $\bar{f}: L \rightarrow L$ by $\bar{f}\left(e_{L}\right)=g^{r}\left(e_{L}\right)$, where $g^{r}\left(e_{L}\right) \in h^{+}\left(f\left(e_{L}\right)\right)$. The construction of $\bar{f}$ ensures that it is well-defined, that it commutes with $g$, and that it is a lifting. If $r$ is even, then $\vec{f}$ is order-preserving. Let $r$ be odd, and suppose that $g^{k}\left(e_{L}\right)<g^{2}\left(e_{L}\right) ;$ then $f g^{k}\left(e_{L}\right) \leq g f^{l}\left(e_{L}\right)$, and

$$
\begin{aligned}
f g^{k}\left(e_{L}\right)=g^{k} f\left(e_{L}\right)=g^{k} h g^{r}\left(e_{L}\right) & =g^{r} g^{k} h\left(e_{L}\right) \\
& \geq g^{r} g^{2} h\left(e_{L}\right)=g^{2} h g^{r}\left(e_{L}\right)=g^{2} f\left(e_{L}\right)=f g^{2}\left(e_{L}\right) .
\end{aligned}
$$

Thus $g^{k} f\left(e_{L}\right)=g^{2} f\left(e_{L}\right)$, and hence $\operatorname{Card}(Z)$ divides $Z-k$. But since $g^{k}\left(e_{L}\right)<g^{l}\left(e_{L}\right)$, it follows from the remark after Lemma 2.16 , that $z-k$ is odd, and so by Lemma 2.16 (i), $Z$ is an antichain. An application of Lemma 4.9 now completes the proof.

COROLLARY 4.11. Let $Z \in G M(X)$, and let $h$ in $S(X, Z)$ be onto. If $f \in S(X, Z)$ and $f(X)=L_{2}$, then $f$ lifts to a map $\bar{f}$ in $S(X, X)$. 
Proof. Let $f_{n}=\left.f\right|_{X_{n}}$. Since $X_{n}=L$ is projective, $f_{n}$ lifts to an S-morphism. $\bar{f}_{n}: x_{n} \rightarrow x_{n} \subseteq X$. Define $\bar{f}: X \rightarrow X$ by $\bar{f}(e)=g^{-n} \bar{f}_{n} g^{n}(e)$, and note that in the loop, $g^{-n}$ is actually (congruent to) a positive power of $g$, so $\bar{f}$ is well-defined. It is constructed to commute with $g$, and is a lifting since

$$
\begin{aligned}
h \bar{f}(e)=h g^{-n} \bar{f}_{n} g^{n}(e)=g^{-n} h \bar{f}_{n} g^{n}(e)=g^{-n} f_{n} g^{n}(e) & \\
& =g^{-n} f g^{n}(e)=g^{-n} g^{n} f(e)=f(e) .
\end{aligned}
$$

Finally, to see that $\bar{f}$ is order-preserving, we check two cases; if $n$ is even, then

$$
\begin{aligned}
g^{k}(e)<g^{l}(e) & \Rightarrow g^{k+n}(e) \leq g^{Z+n}(e) \\
& \Rightarrow \bar{f}_{n} g^{k+n}(e) \leq \bar{f}_{n} g^{Z+n}(e) \\
& \Rightarrow g^{-n-\bar{f}_{n} g^{k+n}}(e) \leq g^{-n} \bar{f}_{n} g^{Z+n}(e) \\
& \Rightarrow \bar{f}^{k}(e) \leq \bar{f} g^{l}(e)
\end{aligned}
$$

and if $n$ is odd, then

$$
\begin{aligned}
g^{k}(e)<g^{l}(e) & \Rightarrow g^{k+n}(e) \geq g^{l+n}(e) \\
& \Rightarrow \bar{f}_{n} g^{k+n}(e) \geq \bar{f}_{n} g^{l+n}(e) \\
& \Rightarrow g^{-n} \bar{f}_{n} g^{k+n}(e) \leq g^{-n} \bar{f}_{n} g^{l+n}(e) \\
& \Rightarrow \bar{f}^{k}(e) \leq \bar{f} g^{l}(e) .
\end{aligned}
$$

COROLLARY 4.12. If $X_{1}$ is the Zoop, then $X$ is projective in $G M(X)$

Proof. Let $Z \in G M(X)$; since $X_{1}$ is the loop, it is not maximal by Theorem 2.14, and so $G M(X)=M(X)$; that is, it is sufficient to show that $X$ is self-projective. Let $f, h \in S(X, 2)$, and let $h$ be onto. Since $X_{1}$ is the loop, $h\left(x_{1}\right)=L_{2}$ the loop of $Z$, so $Z$ is either a loop, or has a one-element tail. If $f(x)=L_{Z}$, then $f$ lifts by Corollary 4.11; and if $f(X) \neq L_{2}$, then $f(X)=2=h(X)$. Thus $f=h$ 
and the identity map on $X$ lifts $f$.

Note that if $X$ is a one-element space, then it is trivially profective in $G M(X)$. If $\operatorname{Card}(X)=2$, then $X$ is the loop or $X_{1}$ is the loop, and again by Corollaries 4.11 and $4.12, X$ is projective in $\mathrm{GM}(X)$.

THEOREM 4.13. Let $\operatorname{Card}(X) \geq 3$ and suppose $X$ is not the loop; if $X_{1}$ is not maximal, then $X$ is projective in $\mathrm{GM}(X)$.

Proof. We use the characterization of maximality given in Theorem 2.14. First if $X_{1}$ is the loop, then we have shown in Corollary 4.12 that $X$ is projective. Otherwise, since $G M(X)=M(X)$, we show that $X$ is self-projective; so let $Z \in \mathrm{GM}(X)$ and let $f, h \in \mathrm{S}(X, Z)$, with $h$ onto. Define $\bar{f}: X \rightarrow X$ by $\bar{f}(e)=g^{r}(e)$ where $h g^{r}(e)=f(e)$. As we have already seen, $\bar{f}$ is well-defined, $\bar{f}$ commutes with $g$, and $h \bar{f}=f$. It remains to be shown that $\bar{f}$ is order-preserving. If $X$ is unordered, then we are done; if $X$ has some non-trivial order relationships then consider the two cases:

(i) There exists $k$ in $N$, with $g^{k}(e) \# g^{k+1}(e)$. Without loss of generality, assume $g^{k}(e)<g^{k+1}(e)$. By Theorem 2.14 (iii), $g^{k+1}(e)=g^{k+2}(e)$. Thus $\bar{f} g^{k}(e)=g^{k+r}(e)=g^{k+r+1}(e)=\bar{f} g^{k+1}(e)$, whence $\bar{f}$ is order-preserving; or $r=0$, whence $h(e)=f(e)$, so $h=f$ and the identity map on $X$ lifts $f$.

(ii) For each $k$ in $N, g^{k}(e) \| g^{k+1}(e)$. Let $g^{s}(e)<g^{t}(e)$. By Theorem 2.14 (iv), this is the only non-trivial order relationship, thus if $\bar{f}_{g}^{s}(e) \neq \bar{f}_{g}^{t}(e)$, then $g^{s+r}(e)>g^{t+r}(e)$, and therefore $g^{t+r}(e)=g^{s}(e)$ and $g^{s+r}(e)=g^{t}(e)$. Now since $g$ is order-reversing, and $g^{s+r}(e) \neq g^{t+r}(e)$, it follows that $g^{s+1}(e)>g^{t+1}(e)$. Hence $g^{t+1}(e)=g^{s}(e)$, giving $g^{t+1}(e)<g^{t}(e)$, which contradicts the hypothesis (ii).

THEOREM 4.14. Let $\operatorname{Card}(X) \geq 3$ and suppose that $X_{1}$ is maximal. If one of the following conditions hold, then $X$ is projective in $G M(X)$. 
(i) $e \# g^{i}(e)$ for some $i>0$.

(ii) $e \| g^{i}(e)$ for each $i>0$, and there exists $k$ and $i$, with $g^{k}(e)<g(e)<g^{l}(e)$.

Note that in condition $(i i), X$ is obtained by adding a new end element to the tail of $X^{\prime}$, where $X^{\prime}$ is the space described at the end of Section 2 .

Proof. Let $Z, W \in G M(X)$, and let $f \in S(X, Z)$ and $h \in S(W, Z)$, with $h$ onto. Define $\bar{f}: X+W$ by $\bar{f}(e)=g^{r}\left(e_{W}\right)$ where $h g^{r}\left(e_{W}\right)=f(e)$. As before, it is trivial to check that $\bar{f}$ is welldefined, commutes with $g$, and is a lifting. As a consequence of the remarks following Theorem 2.12, $W$ is an S-morphic image of either $X$ or $x_{1}$. If it is an image of $x$, then $\bar{f}$ is order-preserving if $r$ is even; and if it is an image of $x_{1}$, then $\bar{f}$ is order-preserving if $r$ is odd.

Otherwise, $\bar{f}$ is order-reversing, in which case

$$
\begin{aligned}
g^{s}(e)<g^{t}(e) & \Rightarrow \bar{f} g^{s}(e) \geq \bar{f} g^{t}(e) \\
& \Rightarrow h \bar{f} g^{s}(e) \geq h \bar{f} g^{t}(e) \\
& \Rightarrow f g^{s}(e) \geq f g^{t}(e) .
\end{aligned}
$$

But since $f$ is order-preserving, $g^{s} f(e)=g^{t} f(e)$. In case $(i)$, $e \# g^{i}(e)$, so $f(e)=g^{i} f(e)$; that is, $f(x)=L_{Z}$ and so $f$ lifts by Corollary 4.11. In case (ii), $g^{k}(e)<g(e)<g^{l}(e)$, so $g^{k} f(e)=g f(e)=g^{2} f(e)$; that is, $f\left(x_{1}\right)=L_{Z}$. Now, if $r>0$, then $g^{k+r-1}(e) \# g^{r}(e) \# g^{l+r-1}(e)$, with the two comparability relations going in the same direction. So without loss of generality, assume that $g^{k+r-1}(e) \leq g^{r}(e) \leq g^{l+r-1}(e)$. Thus $h g^{k+r-1}(e) \leq h g^{r}(e) \leq h g^{l+r-1}(e)$, and since $h g^{r}(e)=f(e)$, we have $f g^{k-1}(e) \leq f(e) \leq g f^{l-1}(e)$. But from Lemma 2.21, we can deduce that $g^{k-1}(e) C g^{l-1}(e)$, so $f g^{k-1}(e)=g f^{l-1}(e)$. 
Therefore $f(e) \in L_{Z}$, whence $f(X)=L_{Z}$ and again we are done by Corollary 4.11. If $r=0$, then $h=f$ and $f$ lifts to the identity map on $X$.

THEOREM 4.15. Let $\operatorname{Card}(X) \geq 3$; then $X$ is projective in $\operatorname{GM}(X)$ if and only if any of the following hold:

(i) $X$ is the Zoop;

(ii) $X_{1}$ is not maximaz;

(iii) $X_{1}$ is maximal and $e \# g^{k}(e)$ for some $k>0$;

(iv) $x_{1}$ is maximal, and $e \| g^{i}(e)$ for each $i>0$ and there exist $k, l$ in $N$, with $g^{k}(e)<g(e)<g^{2}(e)$.

Proof. One direction has been proved by Theorems 4.10, 4.13, and 4.14. Conversely, let $X$ be projective, and assume that $X$ is not the loop, $x_{1}$ is maximal, $e \| g^{i}(e)$ for each $i$ in $N$, and $g(e)$ does not Iie between two distinct elements. Let $c_{2}$ and $c^{2}$ be as in Figure 7 .
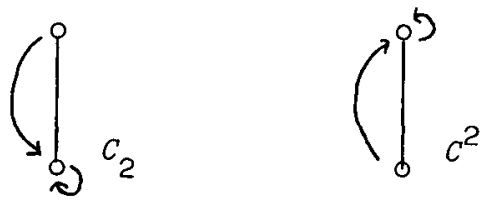

FIGURE 7

If $g(e) \| g^{k}(e)$ for each $k \neq 1$, then both $c_{2}$ and $c^{2}$ are S-morphic images of $x$ and $x_{1}$. If $g(e)<g^{k}(e)$ for some $k$ in $N$, then by assumption $g(e) \ngtr g^{l}(e)$ for all $Z$ in $N$, and it is easily seen that $c^{2}$ is an S-morphic image of both $x$ and $x_{1}$. (If $g(e)>g^{k}(e)$ for some $k$ in $\mathrm{N}$, then dually, we conclude that $C_{2}$ is an S-morphic image of both $X$ and $\left.X_{1} \cdot\right)$ In any case then, both $X$ and $X_{1}$ have a space $C$ in $\operatorname{GM}(X)$ as an S-morphic image. Let $f: X \rightarrow C$ and $h: X_{1} \rightarrow C$ be 
the corresponding surjections. Since $X$ is projective, there is an S-morphism $\bar{f}: X \rightarrow X_{1}$ with $h \bar{f}=f$. But then $h \bar{f}(e)=f(e)=e_{C} ;$ and as $C$ is not a loop and $h$ is onto, we have $\bar{f}(e)=e_{X_{1}}$. But then $X_{I}$ is an S-morphic image of $X$, contradicting the maximality of $X_{1}$.

COROLLARY 4.16. If $x_{1}$ is maximal, then $x_{1}$ is projective in $\operatorname{GM}(X)$ if and only if $X$ is projective in $\operatorname{GM}(X)$.

Proof. Suppose $X$ is projective. Let $Z, W \in \operatorname{GM}(X)$ and let $h \in S(W, z)$ and $f \in S\left(X_{1}, z\right)$ with $h$ onto. Define $\bar{f}: X_{1} \rightarrow W$ as in Theorem 4.14. By the same argument, $\bar{f}$ is either order-preserving or order-reversing. Assume $\bar{f}$ is order-reversing; then by Theorem 4.15, either $e \# g^{k}(e)$ for some $k$ in $N$, or $g^{k}(e)<g(e)<g^{2}(e)$ for some $k, l$ in $N$. In either case, $f\left(x_{1}\right)=L_{z}$ and from Corollary 4.11 we deduce that $f$ lifts; whence $X_{1}$ is projective. The other direction follows easily using a similar argument to the one in Theorem 4.15.

We now have almost enough information to completely characterize injectivity in the subvarieties generated by a single finite algebra. Let $X$ be the dual of a finite subdirectly irreducible distributive Ockham algebra. By applying Proposition $4.1(v)$ and $(v i)$ to Theorem 4.15 and Corollary 4.16, we know when $\operatorname{Var}(O(X))$ has enough injectives, and these results together with Proposition 4.1 (iii) and Theorem 4.10 then tell us exactly which subdirectly irreducible algebras are injective in $\operatorname{Var}(O(X))$.

Furthermore, let $M, A, B \in \mathrm{HS}(O(X))$ with $M$ maximal and suppose that $\phi$ in $O(A, M)$ is onto, and $\psi$ in $O(A, B)$ is one-to-one. If $M \cong O(X)$, then clearly $A \cong O(X)$; and if $M \cong O\left(X_{1}\right)$, then $A \cong O(X)$ or $A \cong O\left(X_{1}\right)$. In either case, $A$ must also be maximal, and so by definition, $\psi$ is an isomorphism. Thus $M$ is weak injective in $H S(O(X))$. We also note that since $O(L)$ is injective in $\operatorname{HS}(O(X)$ ) (by Theorem 4.10), it is weak injective in HS $(O(X))$. Finally, since all weak injectives in a class of algebras are absolute subretracts, Theorem 2.14 tells us exactly which subdirectly irreducible algebras are weak injective in $\operatorname{Var}(O(X))$. 
Now if we can find a simplicity formula for the maximal subdirectly irreducibles we will be done. Let

$$
\alpha(a, b):=a=0 \& b \wedge \sim b=0 \& b \vee \sim b=1 \text {. }
$$

If $U \in O(X)$ and $X-g^{\leftarrow} U=X-U$, then $x \in U$ if and only if $g(x) \in U$. So $U$ is a $g$-subset of $X$. Suppose $U$ is non-empty; that is, $U=x_{k}$ for some $k \leq n$. But then $g\left(g^{k-1}(e)\right)=g^{k}(e) \in U$ and $g^{k-1}(e) \neq U$; so $k=0$. Thus the only elements $b$ of $B=O(X)$, which satisfy $\sim b=b^{\prime}$ are 0 and 1 ; so $\alpha(a, b)$ is actually a simplicity formula for the class of all finite subdirectly irreducible distributive Ockham algebras.

We can now apply Theorem 4.8 to obtain complete descriptions of the weak injectives and injectives in a variety generated by a single finite al gebra.

THEOREM 4.17. Let $X$ be the dual of a finite subdirectly irreducible distributive Ockham algebra, let $K=\operatorname{Var}(O(X))$, and let $A \in \mathrm{K}$.

(a) If $X_{1}$ is not maximal in $\mathrm{GM}(X)$, then the following are equivalent:

(i) $A$ is injective in $K$;

(ii) $A$ is weak injective in $\mathrm{K}$;

(iii) $A \cong O(X)\left[B_{0}\right] \times O(L)\left[B_{1}\right]$, where $B_{0}$ and $B_{1}$ are complete Boolean algebras.

(b) If $X_{1}$ is maximal in $G M(X)$, then $A$ is weak injective in $k$ if and only if $A \cong O(X)\left[B_{0}\right] \times O\left(X_{1}\right)\left[B_{1}\right] \times O(L)\left[B_{2}\right]$, where $B_{0}, B_{1}$, and $B_{2}$ are complete Boolean algebras.

(c) (i). If $X_{1}$ is maximal in $G M(X)$, and $X$ satisfies condition (iii) or (iv) of Theorem 4.15, then $A$ is injective in $K$ if and only if $A \cong O(X)\left[B_{0}\right] \times O\left(X_{1}\right)\left[B_{1}\right] \times O(L)\left[B_{2}\right]$, where $B_{0}, B_{1}$, and $B_{2}$ are complete Boolean algebras.

(ii) If $x_{1}$ is maximal in $G M(X)$, and $X$ does not satisfy 
conditions ( $i$ ii) and (iv) of Theorem 4.15, then $K$ does not have enough injectives, and $A$ is injective in $K$ if and only if $A \cong O(L)[B]$, where $B$ is a complete Boolean algebra.

COROLLARY 4.18. Let $m>n, n \geq 0 ; \mathrm{P}_{\mathrm{m}, \mathrm{n}}$ has enough injectives, and for $A$ in $\mathrm{P}_{\mathrm{m}, \mathrm{n}}, A$ is injective in $\mathrm{P}_{\mathrm{m}, \mathrm{n}}$ if and only if $A \cong L_{m, n}\left[B_{0}\right] \times L_{m-n, 0}\left[B_{1}\right]$, where $B_{0}$ and $B_{1}$ are complete Boolean algebras.

Let $T=L_{2,0}$, and let 3 be the three-element De Morgan algebra $\{0, a, 1\}$ with $\sim a=a$. We have seen that $M$, the variety of De Morgan algebras is $\operatorname{ISP}\left(L_{2,0}\right)$, and Cignoli [7], has shown that $K 1$, the variety of Kleene algebras is ISP(3). Note that $P(3)$ is the totally ordered two element loop.

COROLLARY 4.19. (i) $M$ has enough injectives, and any De Morgan algebra $A$ is injective in $M$ if and only if $A \cong T[B]$, where $B$ is a complete Boolean algebra.

(ii) $K 1$ has enough injectives and any Kleene algebra $A$ is injective in $\mathrm{K} 1$ if and only if $A \cong 3[B]$, where $B$ is a complete Boolean algebra.

Injective De Morgan and Kleene algebras were first described intrinsically in [7].

\section{References}

[1] Raymond Balbes and Philip Dwinger, Distributive Lattices (University of Missouri Press, Columbia, 1974).

[2] B. Banaschewski, "Injectivity and essential extensions in equational classes of algebras", Proceedings of the Conference on Universal Algebra, October 1969, 131-147 (Queen's Papers in Pure and Applied Mathematics, 25. Queen's University, Kingston, Ontario, 1970).

[3] Joel Berman, "Distributive lattices with an additional unary operation", Aequationes Math. 16 (1977), 165-171. 
[4] A. Bialynicki-Birula, "Remarks on quasi-Boolean algebras", Bull. Acad. Polon. Sci. Cl. III 5 (1957), 615-619.

[5] A. Bialynicki-Birula and H. Rasiowa, "On the representation of quasiBoolean algebras", Bull. Acad. Polon. Sci. Cl. III 5 (1957), 259-261.

[6] A. Bialynicki-Birula and H. Rasiowa, "On constructible falsity in the constructive logic with strong negation", Colloq. Math. 6 (1958), 287-310.

[7] Roberto Cignoli, "Injective De Morgan and Kleene algebras", Proc. Amer. Math. Soc. 47 (1975), 269-278.

[8] William H. Cornish, "Monoids acting on distributive lattices", manuscript.

[9] William H. Cornish and Peter R. Fowler, "Coproducts of De Morgan algebras", BuzZ. Austral. Math. Soc. 16 (1977), 1-13.

[10] Brian A. Davey and Moshe S. Goldberg, "The free p-algebra generated by a distributive lattice", Algebra Universalis 11 (1980), 90-100.

[11] Brian A. Davey and Heinrich Werner, "Injectivity and Boolean powers", Math. Z. 166 (1979), 205-223.

[12] Alan Day, "Injectivity in equational classes of algebras", Canad. J. Math. 24 (1972), 209-220.

[13] J. Dugundji, Topology, 9th printing (Allyn and Bacon, Boston, Massachusetts, 1975.

[14] John B. Fraleigh, A first course in abstract algebra (Addison-Wesley, Reading, London, Don Mills, 1967. 2nd edition, 1976).

[15] Leonard Gillman and Meyer Jerison, Rings of continuous functions (Van Nostrand, Princeton, Toronto, London, 1960).

[16] George Grätzer, Lattice theory. First concepts and distributive Zattices (Freeman, San Francisco, 1971).

[17] Bjarni Jónsson, "Algebras whose congruence lattices are distributive", Math. Scand. 21 (1967), 110-121.

[18] J.A. Kalman, "Lattices with involution", Trans. Amer. Math. Soc. 87 (1958), 485-491. 
[19] G.C. Moisil, "Recherches sur l'algèbre de la logique", Ann. Sci. Univ. Jassy 22 (1936), 1-118.

[20] Antonio Monteiro, "Matrices de Morgan caractéristiques pour le calcul propositional classique", An Acad. Brasil Ci. 52 (1960), 1-7.

[21] H.A. Priestley, "Representation of distributive lattices by means of ordered Stone spaces", Buzl. London Math. Soc. 2 (1970), $186-190$.

[22] H.A. Priestley, "Ordered topological spaces and the representation of distributive lattices", Proc. London Math. Soc. (3) 24 (1972), 507-530.

[23] Alasdair Urguhart, "Distributive lattices with a dual homomorphic operation", Studia Logica 38 (1979), 201-209.

[24] Russell C. Walker, The Stone-Čech compactification (Ergebnisse der Mathematik und ihrer Grenzgebiete, 83. Springer-Verlag, Berlin, Heidelberg, New York, 1974).

Department of Mathematics,

La Trobe University,

Bundoora,

Victoria 3083,

Australia. 